Supporting information:

\title{
Quantification of spatial and temporal trends in atmospheric mercury deposition across Canada over the past 30 years
}

Sarah L. Roberts ${ }^{1 *}$, Jane L. Kirk ${ }^{1 *}$, Derek C.G. Muir ${ }^{1}$, Johan A. Wiklund ${ }^{2}$, Marlene

S. Evans ${ }^{3}$, Amber Gleason ${ }^{1}$, Allison Tam 1, Paul E. Drevnick 4, 5 , Ashu Dastoor ${ }^{6}$, Andrei Ryjkov ${ }^{6}$, Fan Yang ${ }^{1}$, Xiaowa Wang ${ }^{1}$, Greg Lawson ${ }^{1}$, Martin Pilote ${ }^{7}$, Jonathan Keating 3, Benjamin D. Barst ${ }^{8,5}$, Jason M.E. Ahad 9, Colin A. Cooke 10, 11

${ }^{1}$ Aquatic Contaminants Research Division, Environment and Climate Change Canada, Burlington, Ontario L7R 4A6, Canada

${ }^{2}$ Biology Department, University of Waterloo, Waterloo, Ontario N2L 3G1, Canada

${ }^{3}$ Watershed Hydrology and Ecology Research Division, Environment and Climate Change Canada, Saskatoon, SK S7N 3H5, Canada

${ }^{4}$ Alberta Environment and Parks, 3535 Research Road NW, Calgary, Alberta T2L 2K8, Canada

${ }^{5}$ Institut national de la recherche scientifique, Centre Eau Terre Environment, 490 rue de la Couronne, Québec, Québec G1K 9A9, Canada

${ }^{6}$ Air Quality Research Division, Environment and Climate Change Canada, Québec H9P 1J3, Canada

${ }^{7}$ Aquatic Contaminants Research Division, Environment and Climate Change Canada, Montréal, Québec, H2Y 2E7, Canada

${ }^{8}$ Water and Environment Research Centre, University of Alaska Fairbanks, Fairbanks, Alaska, 99775, United States

${ }^{9}$ Geological Survey of Canada - Québec Division Québec G1K 9A9, Canada

${ }^{10}$ Department of Earth and Atmospheric Sciences, University of Alberta, Edmonton, Alberta T6G 2E3, Canada

${ }^{11}$ Alberta Environment and Parks, 9888 Jasper Ave, Edmonton, Alberta T5J 5C6, Canada

* Corresponding authors

Correspondence: Jane Kirk (jane.kirk@ec.gc.ca) and Sarah Roberts (sarahlilyroberts@ gmail.com)

For submission to Environmental Science \& Technology

Description of Supporting Information:

Number of pages: 45

Number of figures: 13

Number of tables: 10

References

To adhere to the FAIR (Findability, Accessibility, Interoperability, and Reuse) data principles a raw data file has been uploaded with this manuscript. 


\section{S.1. Site description and sample collection}

Lake sediment cores from 82 lakes across Canada and southern Alaska were collected from 19 regions located nearby $(<100 \mathrm{~km})$ known point source regions including: 1 . Trail British Columbia $(\mathrm{n}=10) 2$. Flin Flon Manitoba ( $n=9)$ 3. Thompson Manitoba $(n=4)$, 4. central Alberta in the periphery of Edmonton and nearby Wabamun ( $n=4), 5$. the Athabasca Oil Sands Region (AOSR; $n=20), 6$. Niagara-HaldimandNorfolk region, Ontario $(n=2)$, 7. Greater Toronto Area, Ontario $(n=2), 8$. Greater Montréal Area, Québec (n=7), 9. Greater Trois Rivières, Québec (n=3) and 10. Greater Québec City Area, Québec $(\mathrm{n}=2)$ and in remote regions (>100 km from point source sites) including 1. southern Yukon $(\mathrm{n}=1) 2$. Prince of Wales Island, Alaska $(n=4), 3$. northern Manitoba $(n=1)$, 4. remote South-central Ontario $(n=1)$ 5. Experimental Lakes Area (ELA), Ontario $(n=5), 6$. remote regions of Québec $(n=3)$ and 7. Kejimkujik National Park, Nova Scotia $(n=4)$ (Table S1, S2). Proximity of each lake to the closest major atmospheric $\mathrm{Hg}$ point source was measured, and lake sites within $100 \mathrm{~km}$ of a point source site were coded ' 1 ' to indicate presence, while those situated $>100 \mathrm{~km}$ of a point source site were coded using ' 0 ' to indicate an absence of a point source. At all sites, small hydrologically simple lake sites were targeted, and sediment cores collected at the deepest depth using a Uwitec gravity corers with 8.6 cm inner diameter acrylic tubes or a Pylonex HTH gravity corer with $7 \mathrm{~cm}$ inner diameter polycarbonate tubes. Data on the coring methods and ${ }^{210} \mathrm{~Pb}$ dating profiles have been published previously for 46 of the 82 lakes in the AOSR, central Alberta, Flin Flon, Thompson, northern Manitoba, ELA, and Nova Scotia. $^{1-6}$

\section{S.2. ${ }^{210} \mathrm{~Pb}$ chronologies}

Sediment core intervals $(0.5-1 \mathrm{~cm}$ intervals) were freeze-dried and homogenised and then dated using ${ }^{210} \mathrm{~Pb}$ methods. ${ }^{210} \mathrm{~Pb}$ chronologies were determined in-house at the ECCC Canada Centre for Inland Waters, Burlington, ON, Institut National de la Recherche Scientifique Eau, Terre, Environment in Québec or at the Flett Research Ltd., Winnipeg, MB using gamma ray spectrometry, ${ }^{137} \mathrm{Cs}$ to validate the ${ }^{210} \mathrm{~Pb}$ models, and the constant rate of supply age $\operatorname{model}^{7}$ (Table S4; Fig. S1).

\section{S.3. Hg analyses}


For each sediment interval, c. $100 \mathrm{mg}$ of sediment was analysed for THg by thermal decomposition amalgamation, and subsequent determination by atomic absorption spectrophotometry following USEPA method 7473. All sediment cores were analysed for THg using a direct mercury analyser (DMA80) at the ECCC Canada Center for Inland Waters, Burlington, ON except for samples from 6 lakes (Scout-1, Control, Mountain Goat, Trumpeter, Cabin, and Hasse Lake) which were analysed as described in Barst et al. ${ }^{5}$ Standard reference materials (SRMs) Tort-2, Mess-3 and 2976 Oyster, were analysed daily to validate the accuracy of the instrument and measurements were within their certified range $\left(270 \pm 60,91 \pm 9\right.$ and $61 \pm 3.6 \mathrm{ng} \mathrm{g}^{-1}$, respectively $)$. Approximately $20 \%$ of all sediment samples were analyzed in duplicate or triplicate and replicates were within $6 \%$ precision of each other.

\section{S.4. Calculations}

Anthropogenic Hg fluxes were calculated for each sediment cores (Fig. S2) using sedimentation rates (Fig. S3).

Anthropogenic $\mathrm{Hg}$ fluxes $\left(\mathrm{HgF}_{\mathrm{FF}}\right)\left(\mu \mathrm{g} \mathrm{m}^{-2} \mathrm{yr}^{-1}\right)$ were calculated as:

$$
=\frac{\left(\mathrm{Hg}_{\mathrm{i}}-\mathrm{Hg}_{\mathrm{bk}}\right) \times \text { Sedimentation rate }}{\mathrm{FF}}
$$

Anthropogenic $\mathrm{Hg}$ inventory $\left(\mathrm{HgI}_{\mathrm{FF}}\right)\left(\mu \mathrm{g} \mathrm{m}^{-2}\right)$ were calculated as:

$$
\left.=\int_{i=t=\text { present }- \text { day }}^{i=t \geq \text { baseline }}\left(\left(\mathrm{Hg}_{\mathrm{i}}-\mathrm{Hg}_{\mathrm{bk}}\right) \mathrm{X} \text { Interval mass } \mathrm{i}\right)\right) / \mathrm{FF}
$$

Where: $\mathrm{Hg}_{\mathrm{i}}=\mathrm{Hg}$ concentration $\left(\mu \mathrm{g} \mathrm{kg}^{-1}\right)$ of sediment interval $i$ and $\mathrm{Hg}_{\mathrm{bk}}$ is the mean $\mathrm{Hg}$ baseline concentration, FF refers to the sediment focussing correction, and (t) is the time period that the sediment intervals cover.

Anthropogenic $\mathrm{Hg}$ fluxes (deposition per year; $\mu \mathrm{g} \mathrm{m}^{-2} \mathrm{yr}^{-1}$ ) were calculated for all 82 lake sediment cores using the period before industrialisation specific to each of the 19 regions as a baseline (Table 
S3). For sediment cores in the Ontario region (Lake Nanticoke, Lake Medad, Windmill Point Pond and Grenadier Pond sites) which did not extend beyond baseline time periods, baseline (pre-1850) Hg concentrations from a Lake Ontario sediment core $\left(43^{\circ} 26^{\prime} 01^{\prime \prime} \mathrm{N}\right.$ and $79^{\circ} 24^{\prime} 00^{\prime \prime} \mathrm{W}$ ) (Fig. S4) were applied. For one sediment core in the western Québec region (Lake Brisebois) which did not extend beyond baseline time periods, baseline (pre-1850) $\mathrm{Hg}$ concentrations from Lake Laflamme and Lake Clair were used. For one sediment core in the Greater Montréal City region (Lake Hertel) which did not extend beyond baseline time periods (pre-1850), Hg concentrations from Lake Bouleaux and Lake Gale were used.

Sediment focussing ( $\mathrm{FF})$ corrections were applied to anthropogenic $\mathrm{Hg}$ fluxes $\left(\mathrm{HgF}_{\mathrm{FF}}\right)$ and inventories $\left(\mathrm{HgI}_{\mathrm{FF}}\right)$ for all 82 lake cores to account for particle focussing at deep points in the lake where cores are collected. Correction for sediment focussing allows depositional fluxes to be compared among lakes as well as to measured and modelled atmospheric deposition. ${ }^{8-10}$ To determine if lakes with larger catchments received proportionally larger inputs of $\mathrm{Hg}$ to the lake sediments, the impacts of catchment area: lake area (CA: LA) ratio on $\mathrm{Hg}$ deposition records were examined. The CA:LA correction accounts for lithogenic inputs of $\mathrm{Hg}$ as well as proportionally larger exports of atmospheric $\mathrm{Hg}$ deposition to the catchment. The CA:LA ratios were calculated for each of the 82 lakes using topographic maps which displayed the water flow direction. ${ }^{11}$ Relationships between calculated anthropogenic $\mathrm{Hg}$ inventories $\left(\mu \mathrm{g} \mathrm{m}^{-2}\right.$ ) (or $\mathrm{Hg}$ total deposition) and CA:LA ratio were then examined for the six sampling regions (Trail British Columbia, Flin Flon Manitoba, Thompson Manitoba, Experimental Lakes Area, Greater Montréal Area, and Kejimkujik National Park) where multiple lakes were cored within a region and dry mass data was available. When a strong, significant relationship $\left(\mathrm{r}^{2}\right.$ $>0.4$ ) between anthropogenic Hg inventories and CA:LA ratio for lakes of a given sampling region was observed, then a catchment effect correction ( $\mathrm{CA}$ ) was applied to anthropogenic $\mathrm{Hg}$ fluxes $\left(\mathrm{HgF}_{\mathrm{FF}, \mathrm{CA}}\right)$ to the cores from that region ${ }^{3}$. This catchment correction factor was calculated by dividing the slope by the intercept of the linear relationship between cumulative anthropogenic $\mathrm{Hg}$ inventory and CA:LA. 


\section{References}

${ }^{1}$ Cooke, C.A., Kirk, J.L., Muir, D.C.G., Wiklund, J.A., Wang, X., Gleason, A., Evans, M.S. 2017. Spatial and temporal patterns in trace element deposition to lakes in the Athabasca oil sands region (Alberta, Canada). Environmental Research Letters, 12, 1-11.

${ }^{2}$ Wiklund, J.A., Kirk, J.L., Muir, D.C.G., Evans, M., Yang, F., Keating, J., Parsons, M.T. 2017. Anthropogenic mercury deposition in Flin Flon Manitoba and the Experimental Lakes Area Ontario (Canada): A multi-lake sediment core reconstruction. Science of the Total Environment, 586, 685 695.

${ }^{3}$ Roberts, S., Kirk, J.L., Wiklund, J.A., Muir, D.C.G., Yang, F., Gleason, A., Lawson, G. 2019. Mercury and metal(loid) deposition to remote Nova Scotia lakes from both local and distant sources. Science of the Total Environment, 675, 192-202.

${ }^{4}$ Wiklund, J.A., Kirk, J.L., Muir, D.C.G., Gleason, A., Carrier, J., Yang, F. 2020. Atmospheric trace metal deposition to remote Northwest Ontario, Canada: Anthropogenic fluxes and inventories from 1860 to 2010. Science of the Total Environment, 749, 1-20.

${ }^{5}$ Barst, B.D., Ahad, J.M.E., Rose, N.L., Jautzy, J.J., Drevnick, P.E., Gammon, P.R., Sanei, H., Savard, M. 2017. Lake-sediment record of PAH, mercury, and fly-ash particle deposition near coal-fired power plants in Central Alberta, Canada. Environmental Pollution, 231, 1, 644-653.

${ }^{6}$ Bérubé Tellier, A., Drevnick, P.E., Bertolo, A. 2016. Brook trout (Salvelinus fontinalis) extinction in small boreal lakes revealed by ephippia pigmentation: a preliminary analysis. Advances in Oceanography and Limnology, 7 (2), 197-205.

${ }^{7}$ Appleby, P.G. 2001. Chronostratigraphic techniques in recent sediments. In: Last, W.M., Smol, J.P. (Eds), Tracking Environmental Change using lake sediments, volume 1: Basin analysis, coring, and chronological techniques. Kluwer Academic Published, Dordrecht, pp. 171-203.

${ }^{8}$ Blais, J.M., Kalff, J. 1995. The influence of lake morphometry on sediment focussing. Limnology and Oceanography, 40, (3), 582-588.

${ }^{9}$ Perry, E., Norton, S.A., Kamman, N.C., Lorey, P.M., Driscoll, C.T. 2005. Deconstruction of historic mercury accumulation in lake sediments, northeastern United States. Ecotoxicology, 14, 85 - 99. 
${ }^{10}$ Muir, D.C.G., Wang, X., Yang, F., Nguyen, N., Jackson, T.A., Evans, M.S., Douglas, M., Köck, G., Lamoureux, S., Pienitz, R., Smol, J.P., Vincent, W.F., Dastoor, A. 2009. Spatial trends and historical deposition of mercury in eastern and northern Canada inferred from lake sediment cores. Environmental Science \& Technology, 43, 4802-4809.

${ }^{11}$ Natural Resources Canada, 2020: https://www.nrcan.gc.ca 12 Canadian Air and Precipitation Monitoring Network (CAPMoN) 2020: https://www.canada.ca/en/environment-climate-change/services/air-pollution/monitoring-networksdata/canadian-air-precipitation.html

${ }^{13}$ Mercury Deposition Network (MDN) 2020: http://nadp.slh.wisc.edu/mdn/

${ }^{14}$ Graydon, J.A., St. Louis, V.L., Hintelmann, H., Lindberg, S.E., Sandilands, K.A., Rudd, J.W.M., Kelly, C.A., Hall, B.D., Mowat, L.D. 2008. Long-term wet and dry deposition of total and methylmercury in the remote boreal ecoregion of Canada. Environmental Science \& Technology, 42, 8345-8351. 


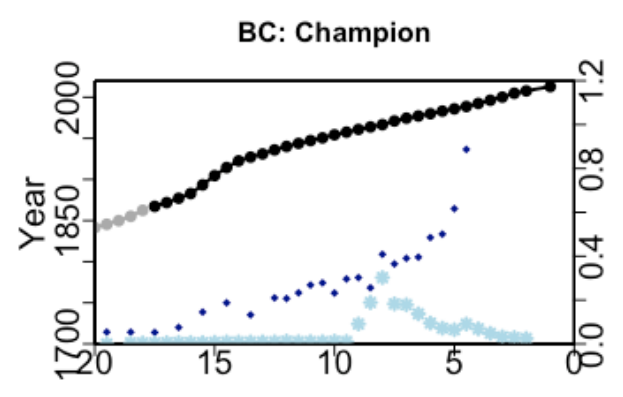

BC: Rosebud

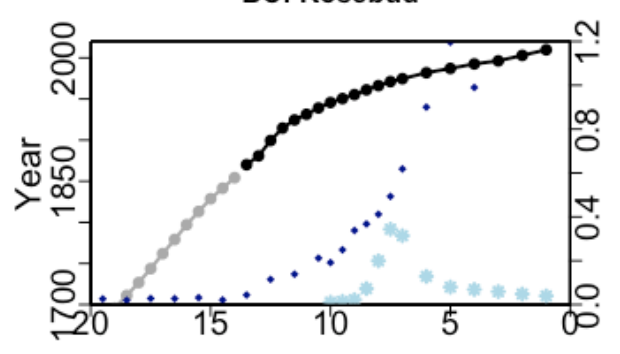

BC: Bridal

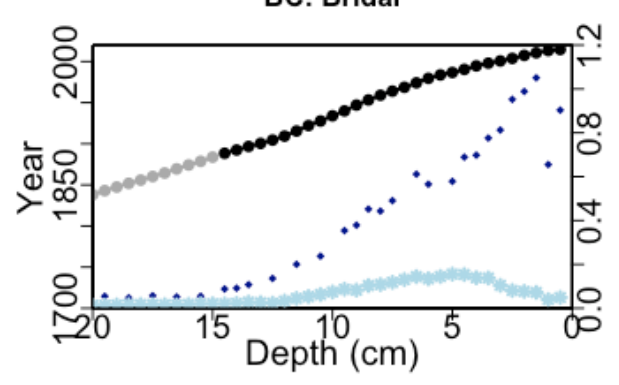

BC: Cottonwood

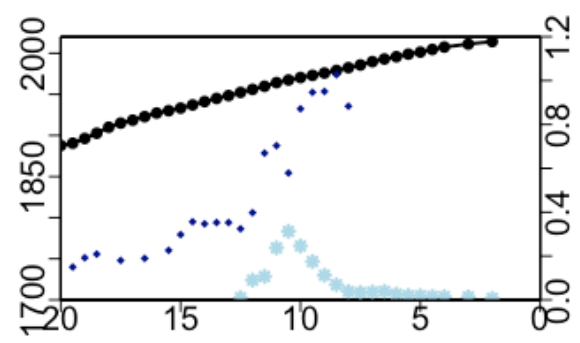

BC: Lomond

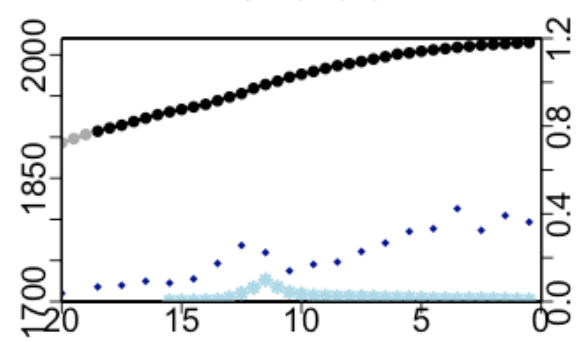

BC: Wilgress

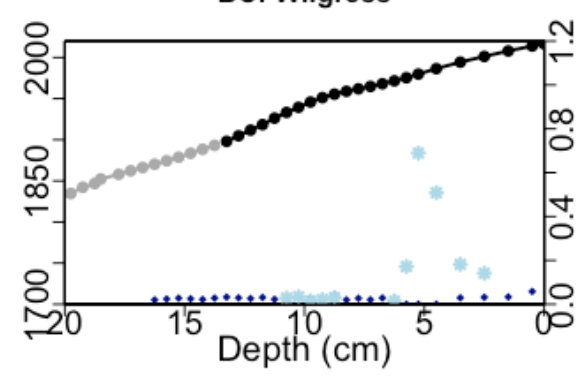

BC: Erie

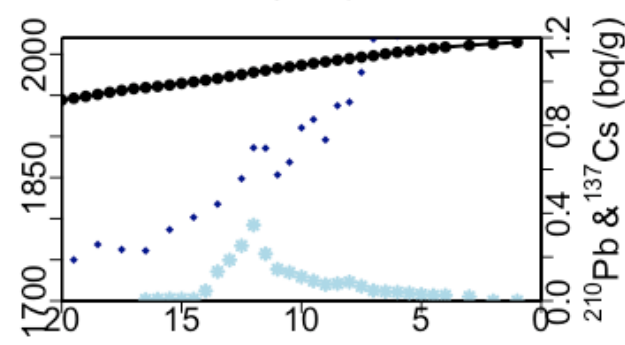

BC: Nancy Greene

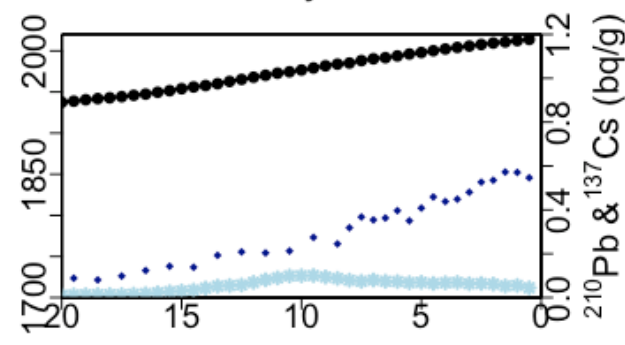

BC: Summit

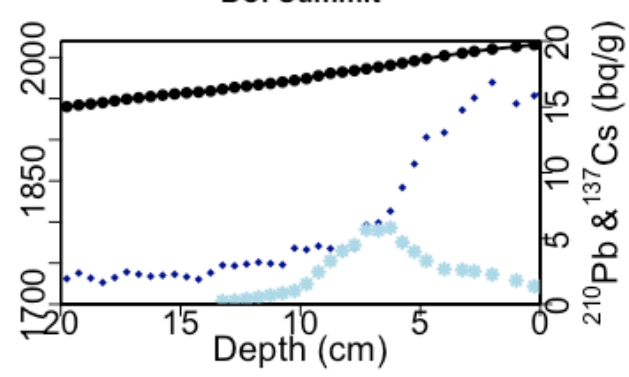

Figure S1. Sediment core chronology, ${ }^{210} \mathrm{~Pb}$ and ${ }^{137} \mathrm{Cs}$ activity (bq/g) profiles for 28 of the 82 lake sediment cores across Canada. ${ }^{210} \mathrm{~Pb}$ dating profiles have been published previously for 46 of the 82 lakes in the Athabasca Oil Sands Region, central Alberta, Flin Flon, Thompson, northern Manitoba, ELA, and Nova Scotia. ${ }^{1-6}$ The black line represents modelled dates using Constant Rate of Supply (CRS) model and the grey lie represents the interpolated dates. 

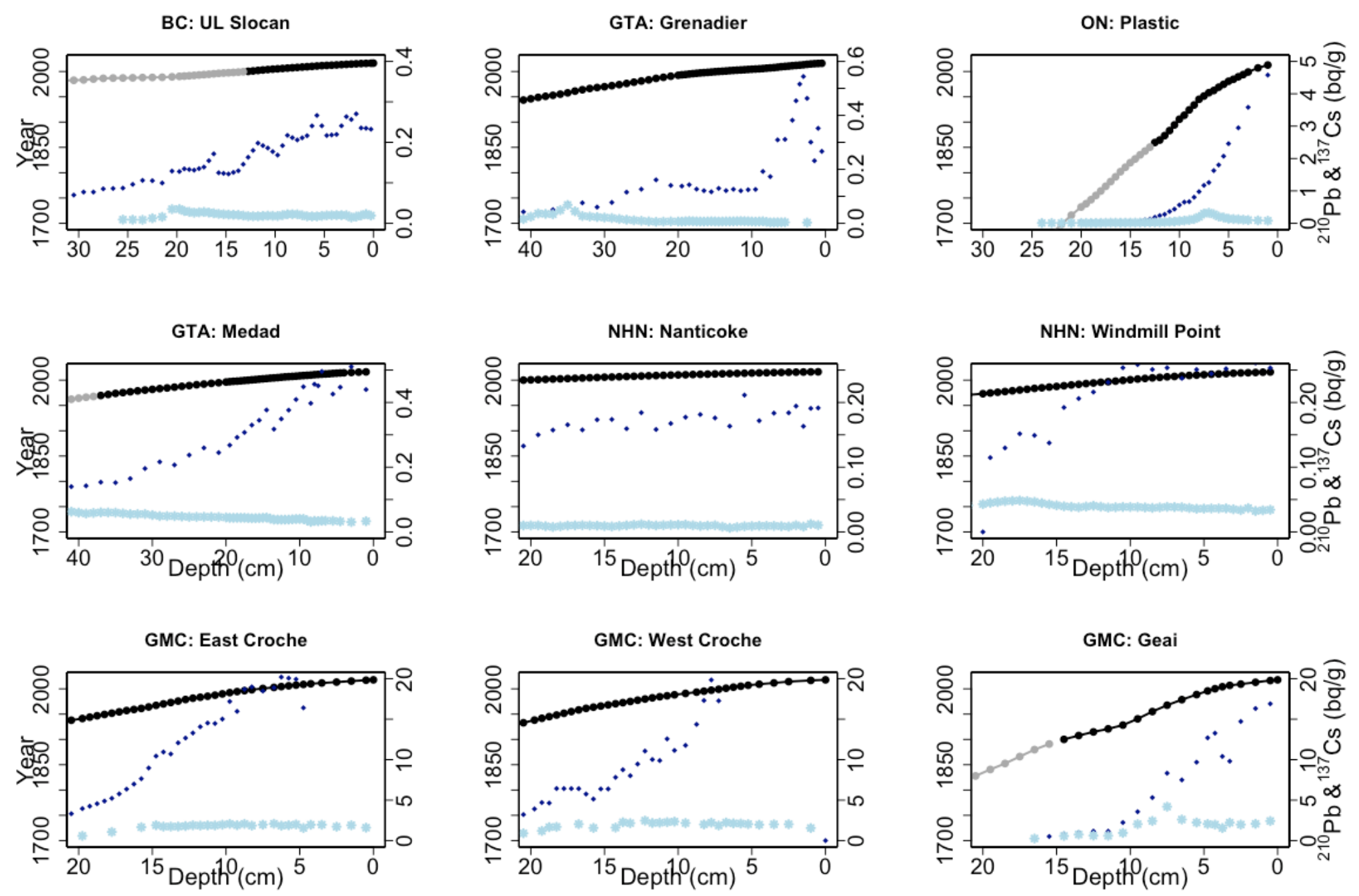


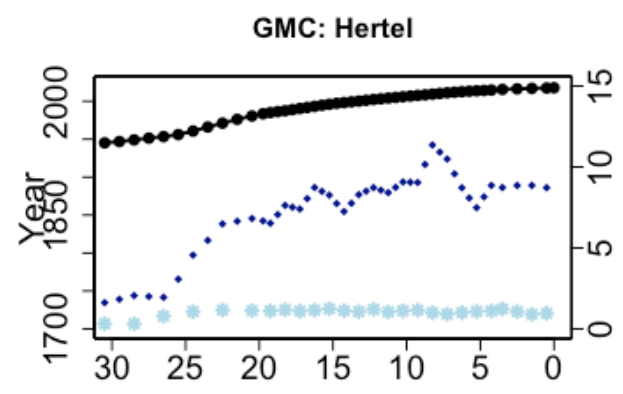

GMC: Gale

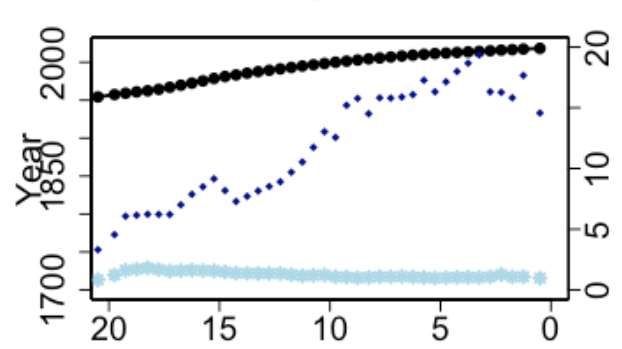

WQC: Brisebois

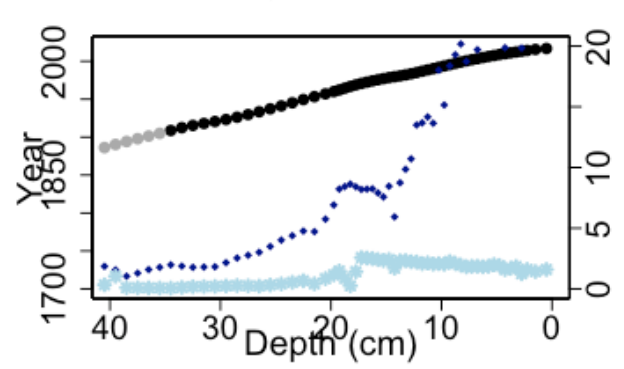

GQC: Clair

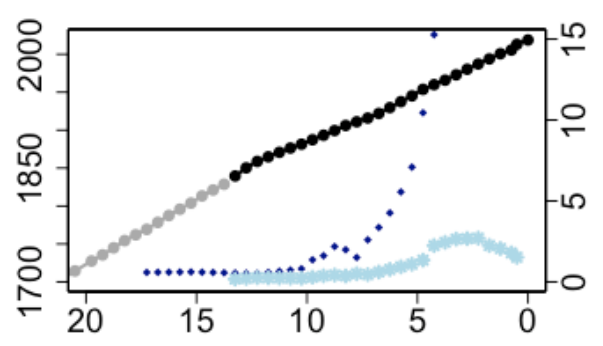

GQC: LaFlamme

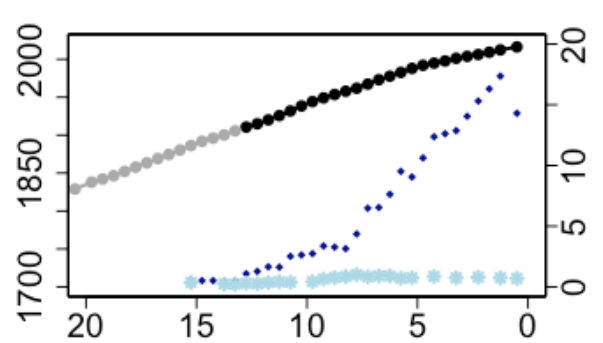

AB: Pigeon

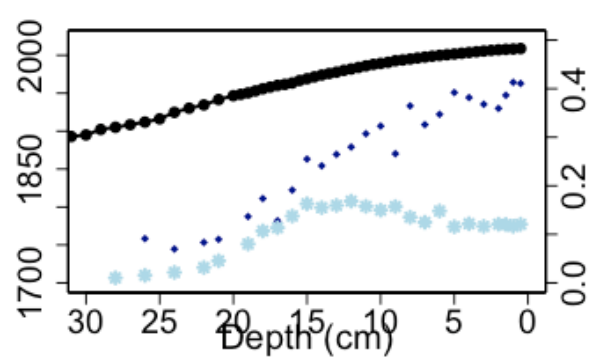

GMC: Bouleaux

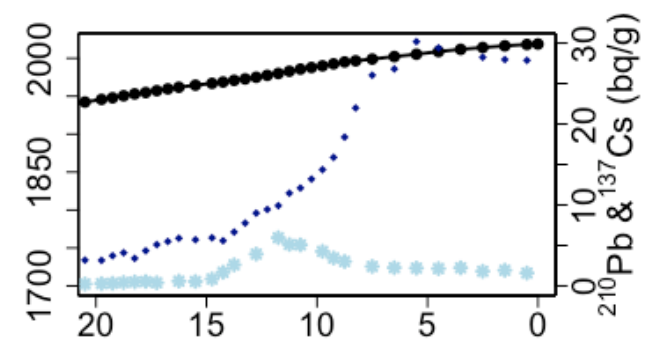

GMC: Triton

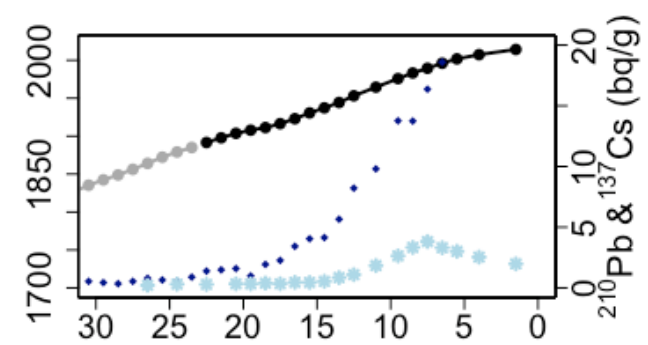

AB: Wolf

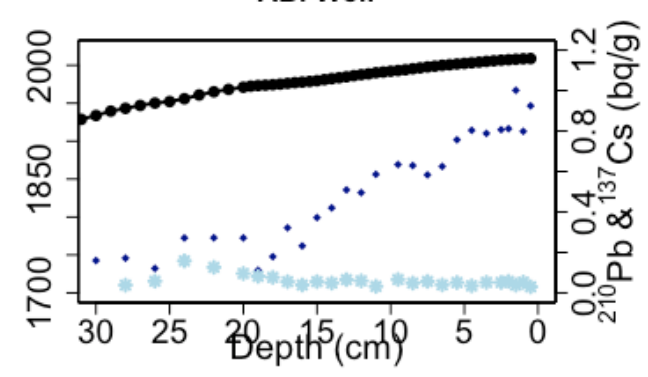



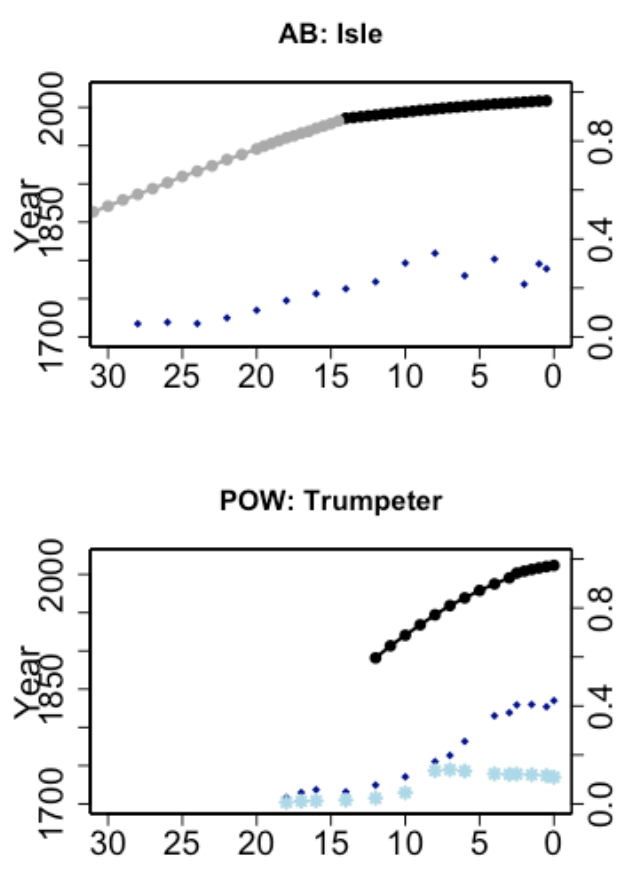

EQC: des lles

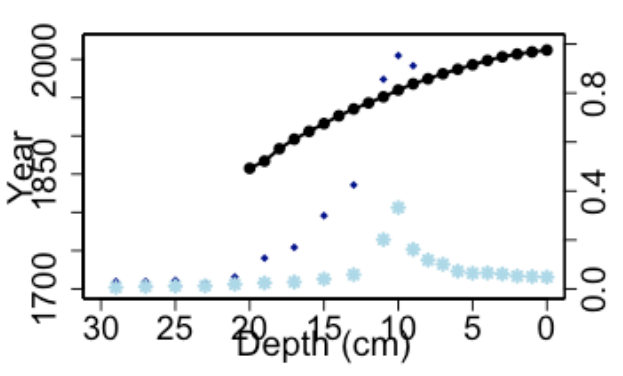

YK: Scout-1

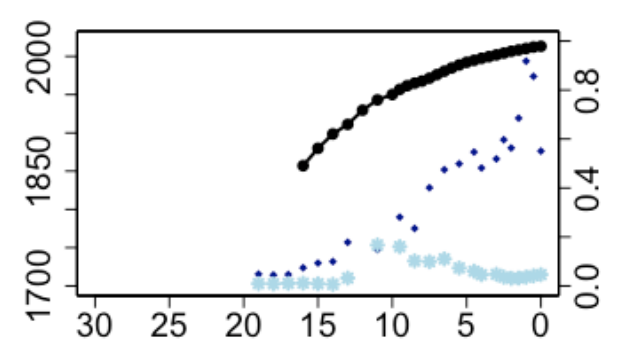

POW: Mountain Goat

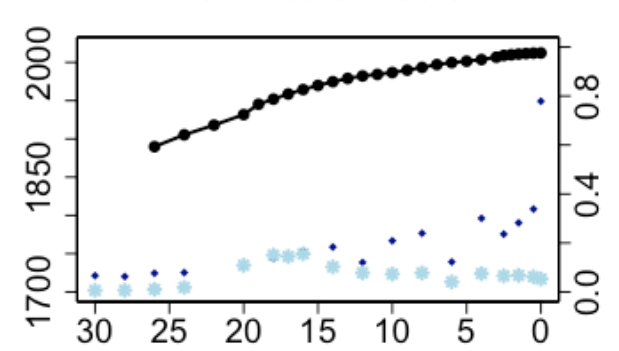

GTR: Francais

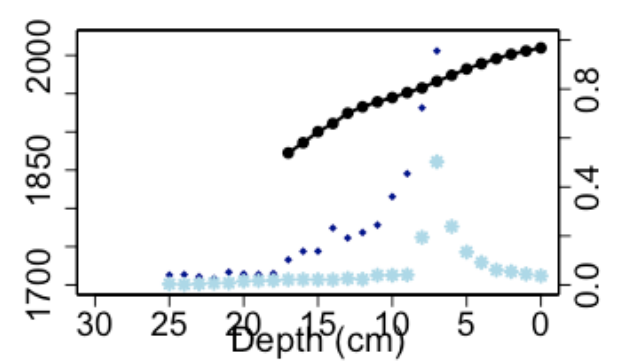

POW: Cabin

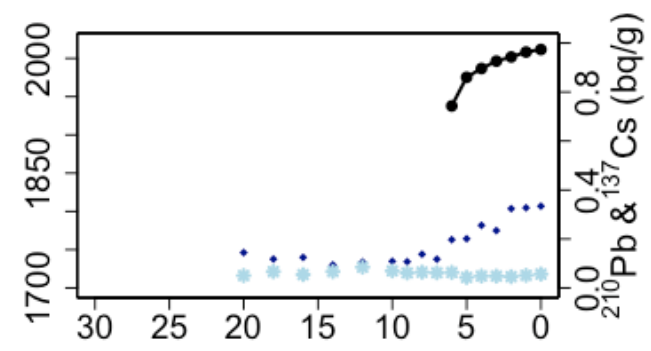

POW: Control

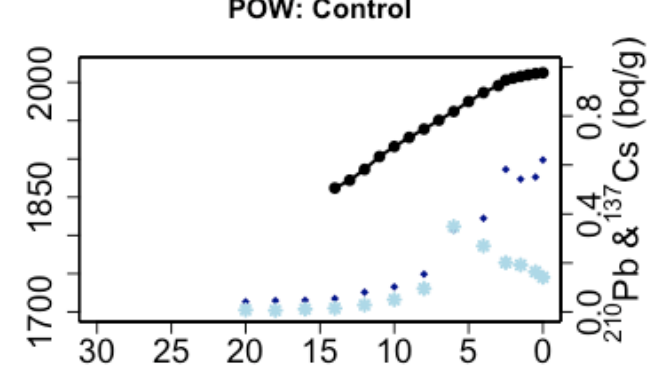



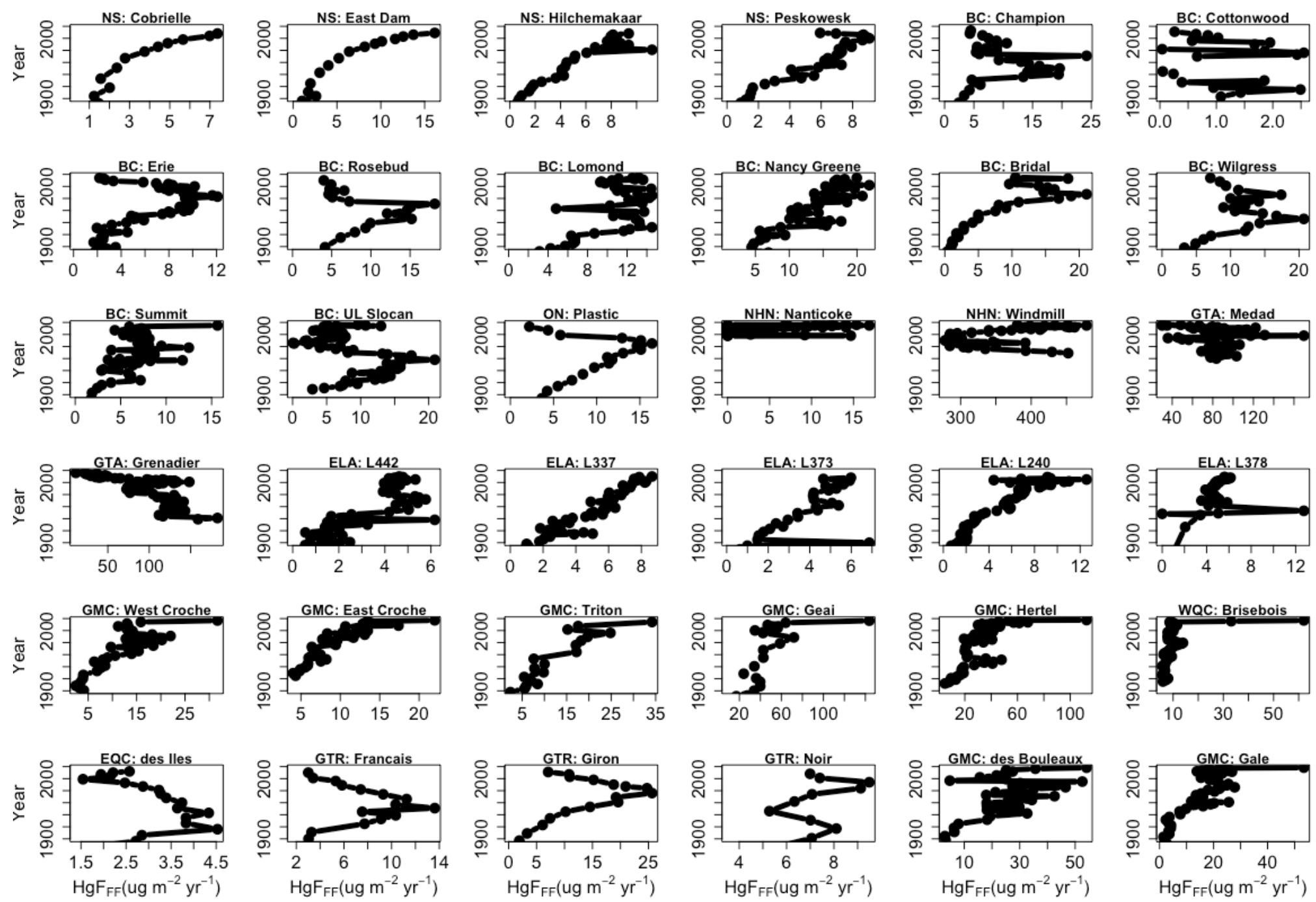

Figure S2. Anthropogenic Hg fluxes $\left(\mathrm{HgF}_{\mathrm{FF}, \mathrm{CA}}\right)$ from 1900-core collection for the 82 lake sediment cores across Canada. The $x$-axis scale varies among the panels. 

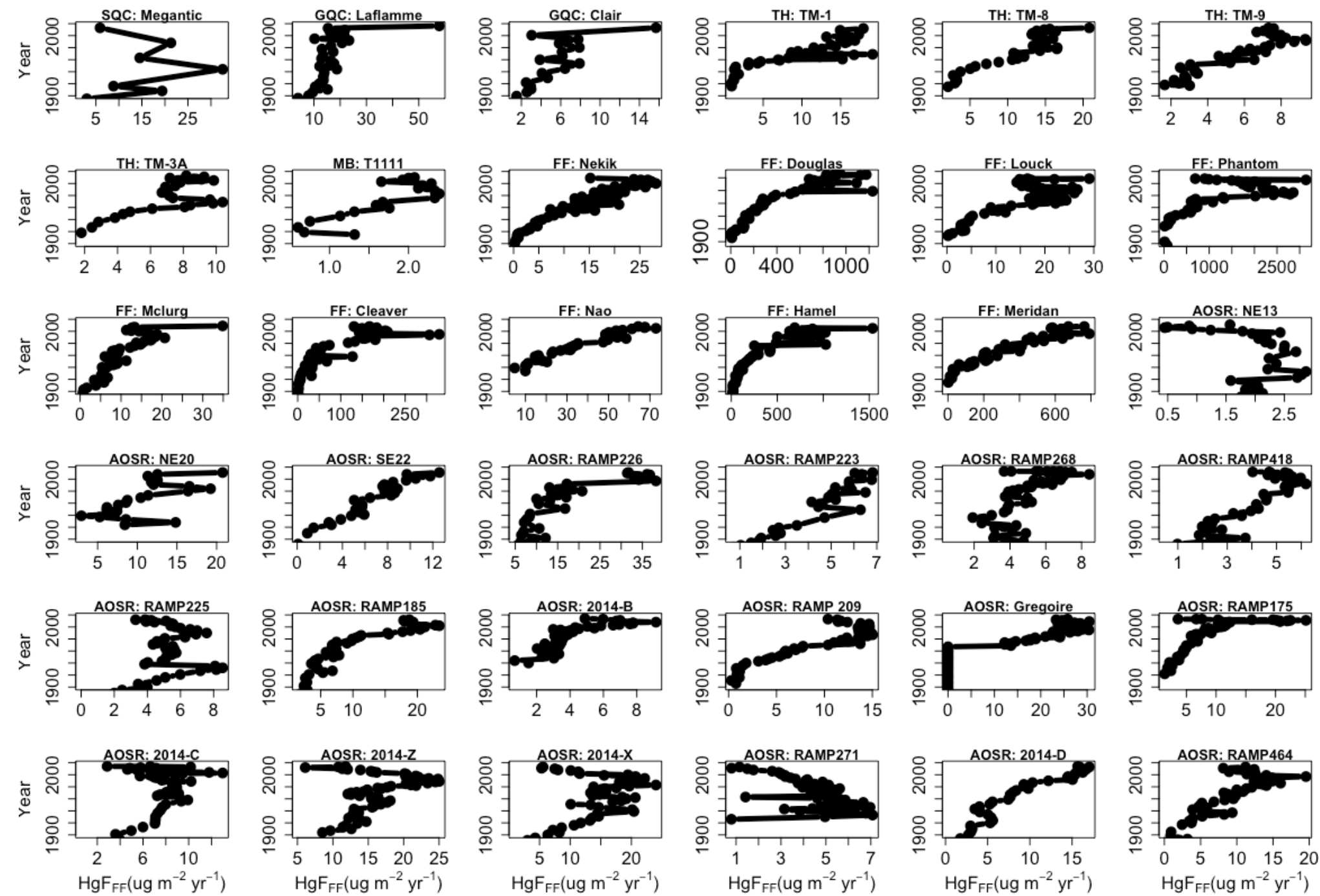

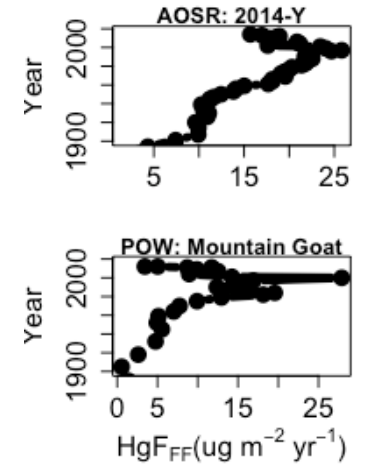
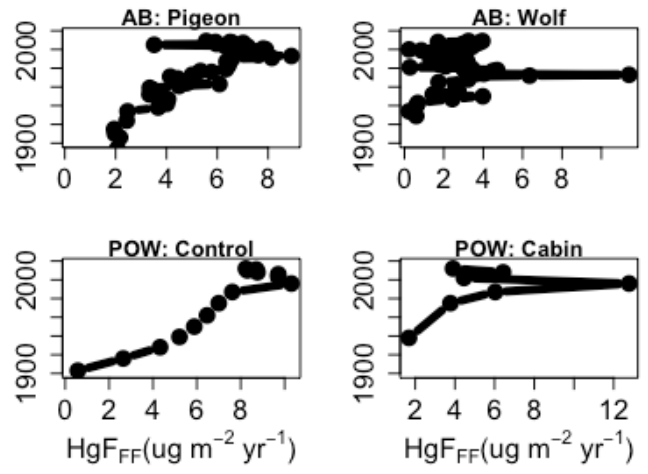
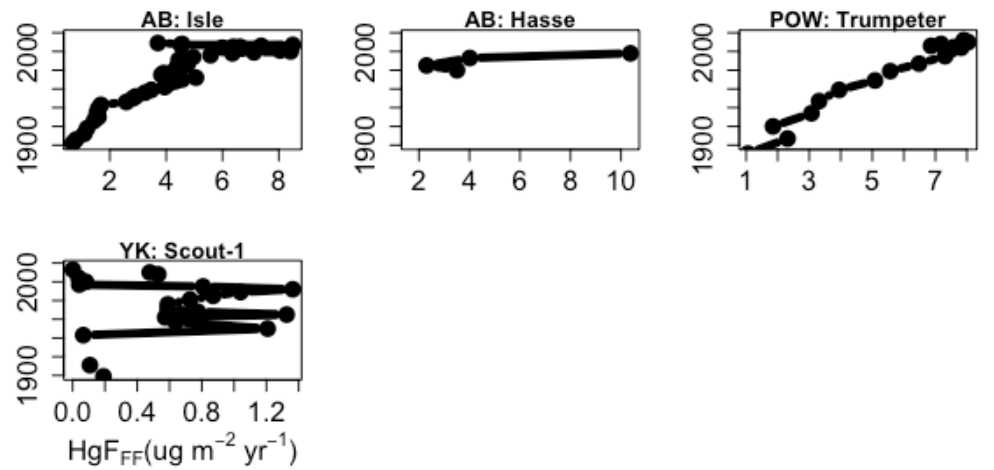

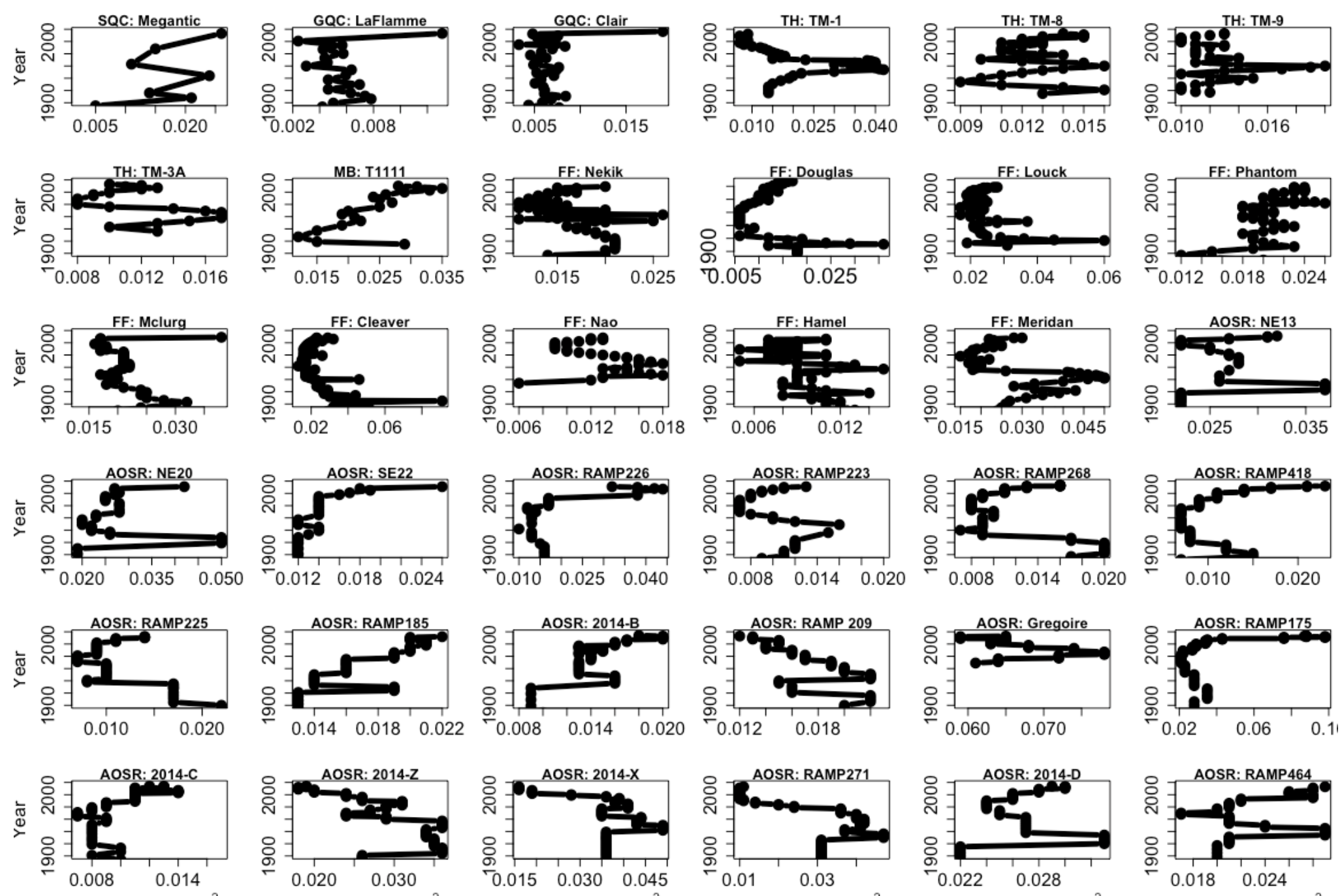

Accumulation $\left(\mathrm{g} \mathrm{cm}^{-2} \mathrm{yr}\right.$

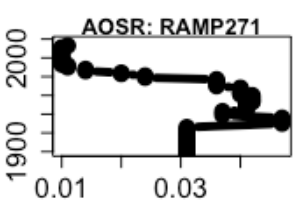

Accumulation $\left(\mathrm{g} \mathrm{cm}^{-2} \mathrm{yr}\right.$
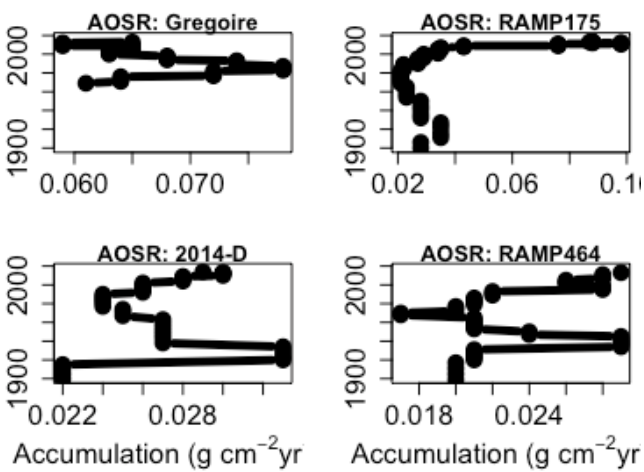

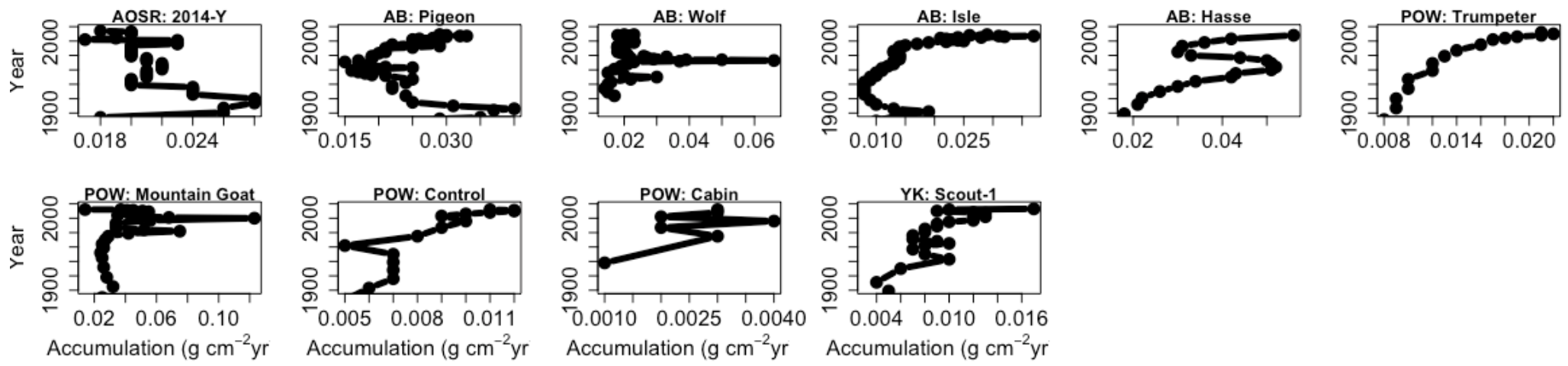


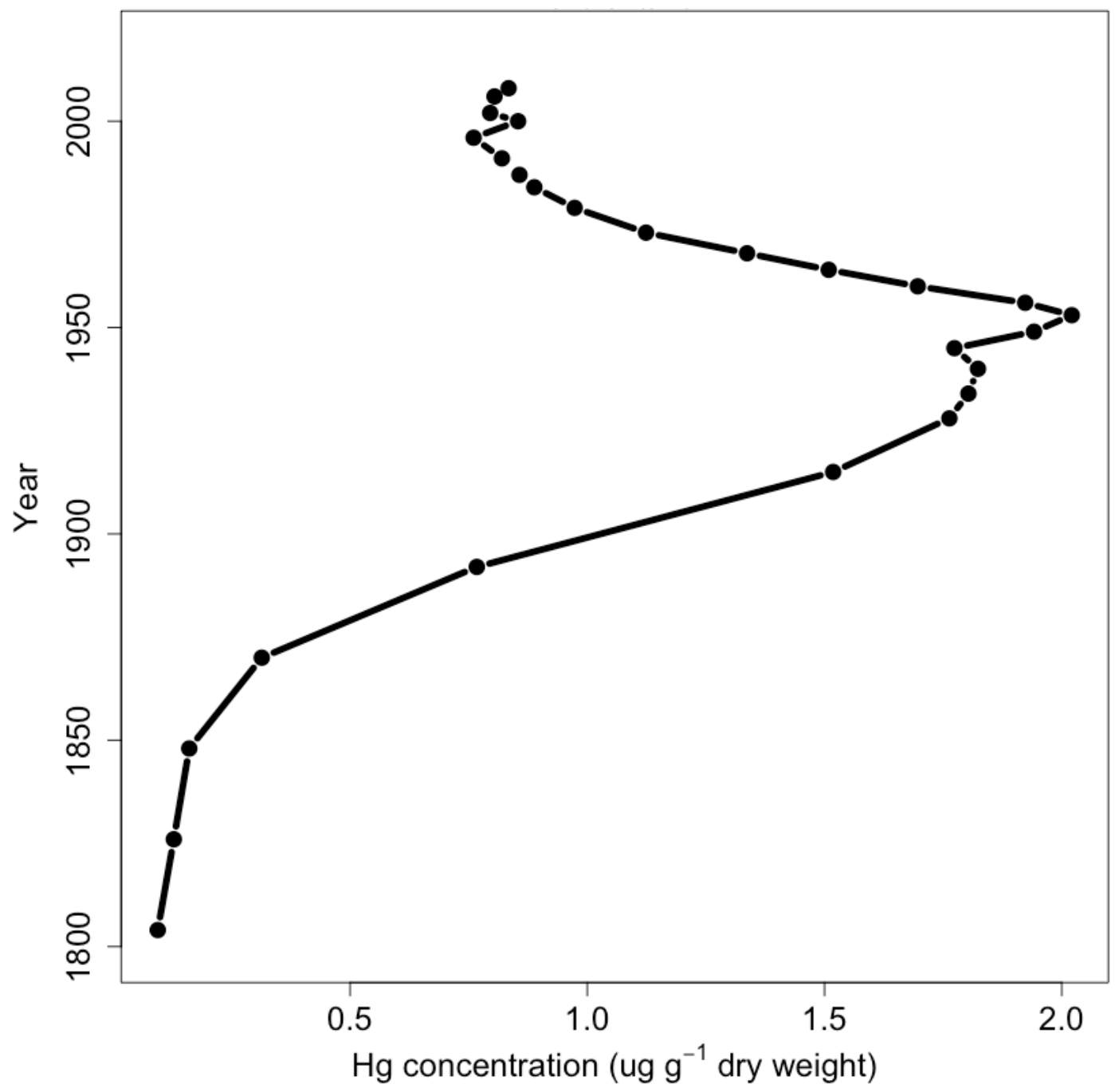

Figure $\mathrm{S4}$. Hg concentrations over time in Lake Ontario sediment core $\left(4^{\circ} 26^{\prime} 01^{\prime \prime} \mathrm{N}\right.$ and $7^{\circ} 24^{\prime} 00^{\prime}$ W) used to calculate pre-industrial/background $\mathrm{Hg}$ concentrations (pre-1850) for the Niagara-Haldimand-Norfolk and Greater Toronto Area lake sediment cores in this study which did not extend beyond baseline time periods (baseline of pre-1850). 


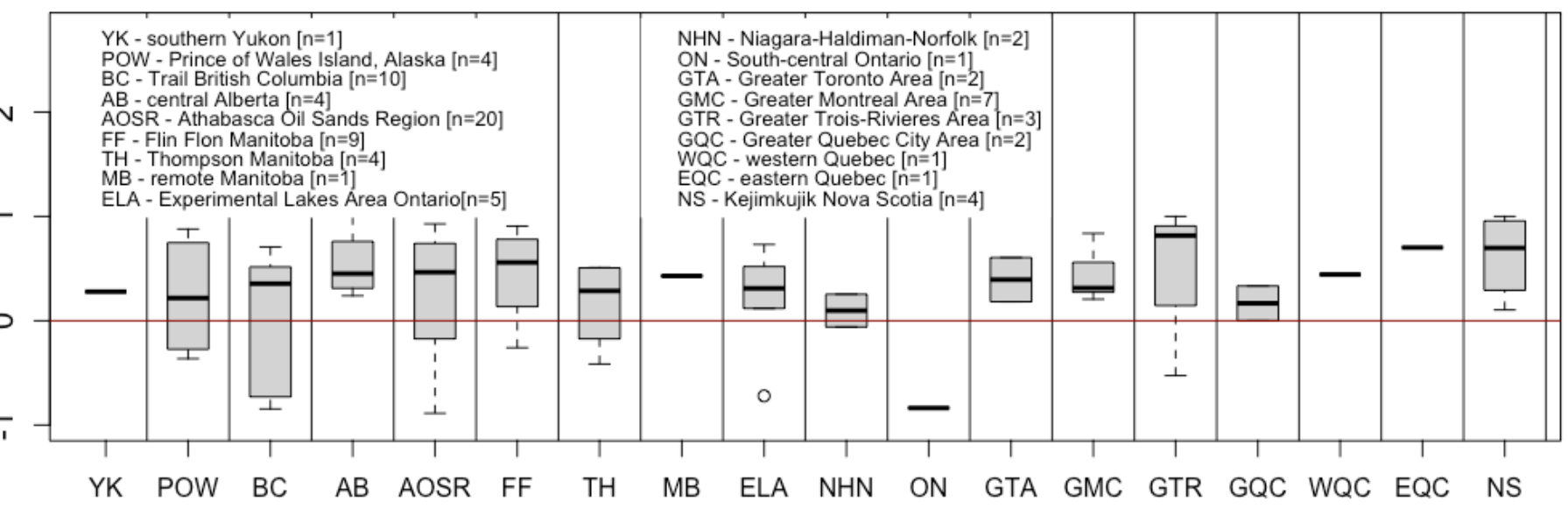

Figure S5. Box and whisker plot showing the median with upper and lower quantiles for trends in post-1990 sedimentation rates for 18 of the 19 study regions across Canada as determined by Mann-Kendall (MK) analyses. A positive (> 0) MK coefficient represents an increasing trend while a negative $(<0)$ MK coefficient represents a decreasing trend. The remote southern Québec region $(n=1)$ is omitted as the core only has one sediment interval covering the post1990 period. 
(A) Sedimentation vs geographic location scatter plots
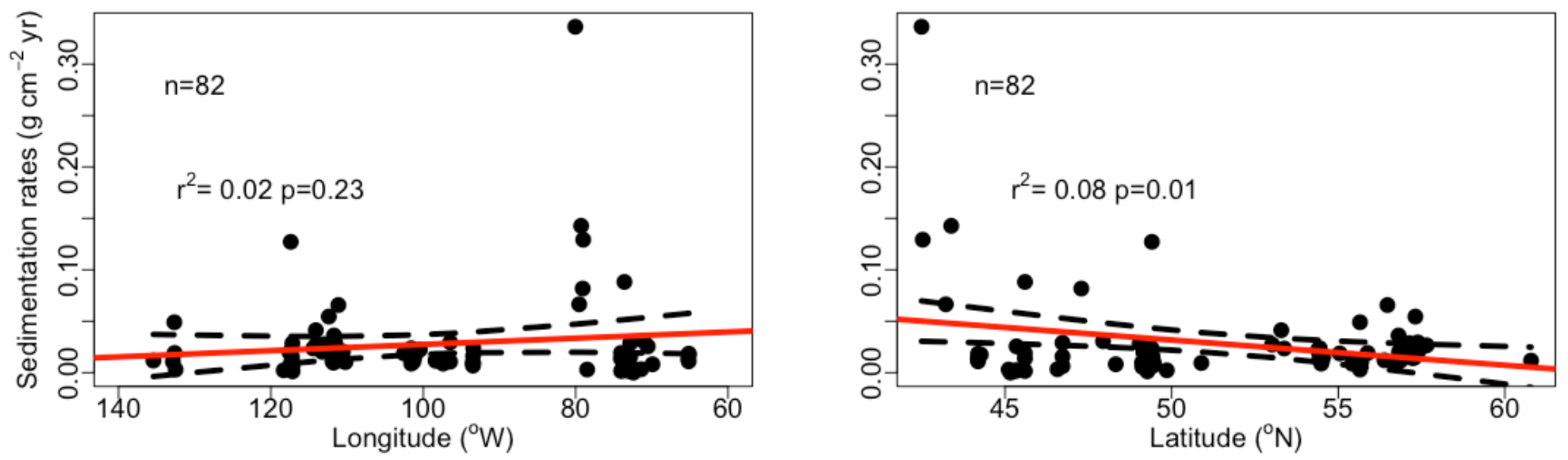

(B) Average regional post-1990 sedimentation rates

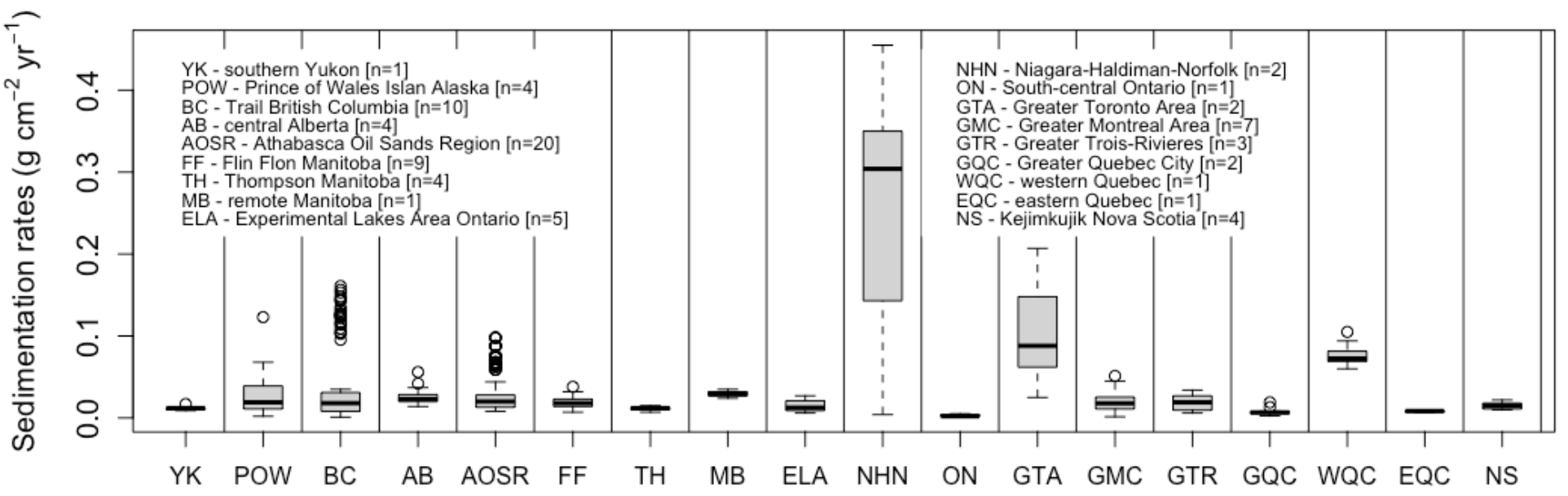

Figure S6. (A) Pearson correlations with linear regression between average post-1990 sedimentation rates and geographical location (longitude and latitude) for 81 study lakes across Canada; (B) Box and whisker plot showing the median with upper and lower quantiles for average post-1990 sedimentation rates in study lakes from 18 of the 19 study regions. The remote southern Québec region $(n=1)$ is omitted as the core only has one sediment interval covering the post-1990 period. 


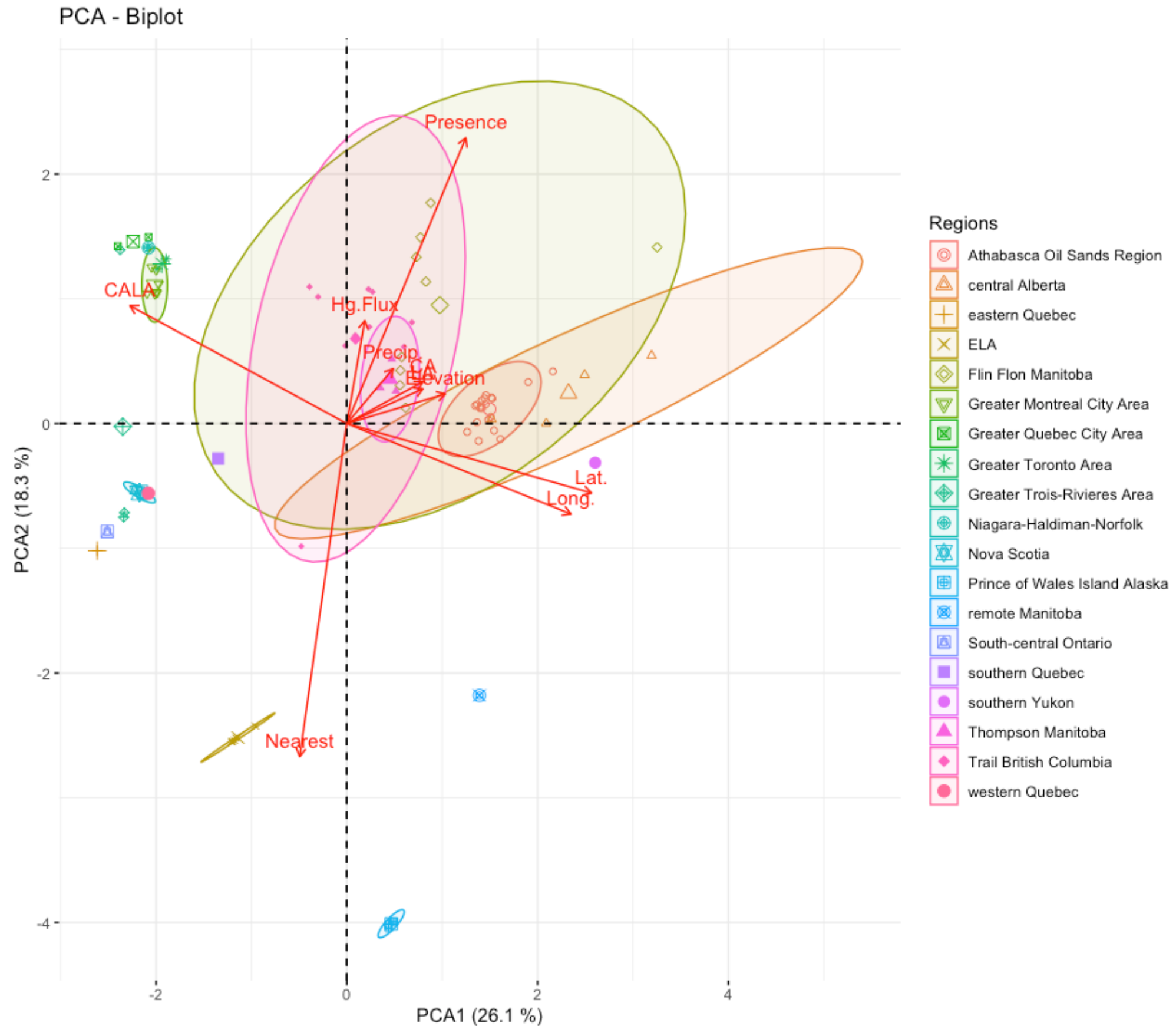

Figure S8. PCA of post-2005 average anthropogenic $\mathrm{Hg}$ fluxes $\left(\mathrm{HgF}_{\mathrm{FF}, \mathrm{CA}}\right)$ and environmental factors; including post-2005 precipitation quantity, lake area (LA), catchment area (CA), lake area: catchment area (CA:LA), elevation, longitude, latitude, distance to nearest point source and presence/absence of point source. 


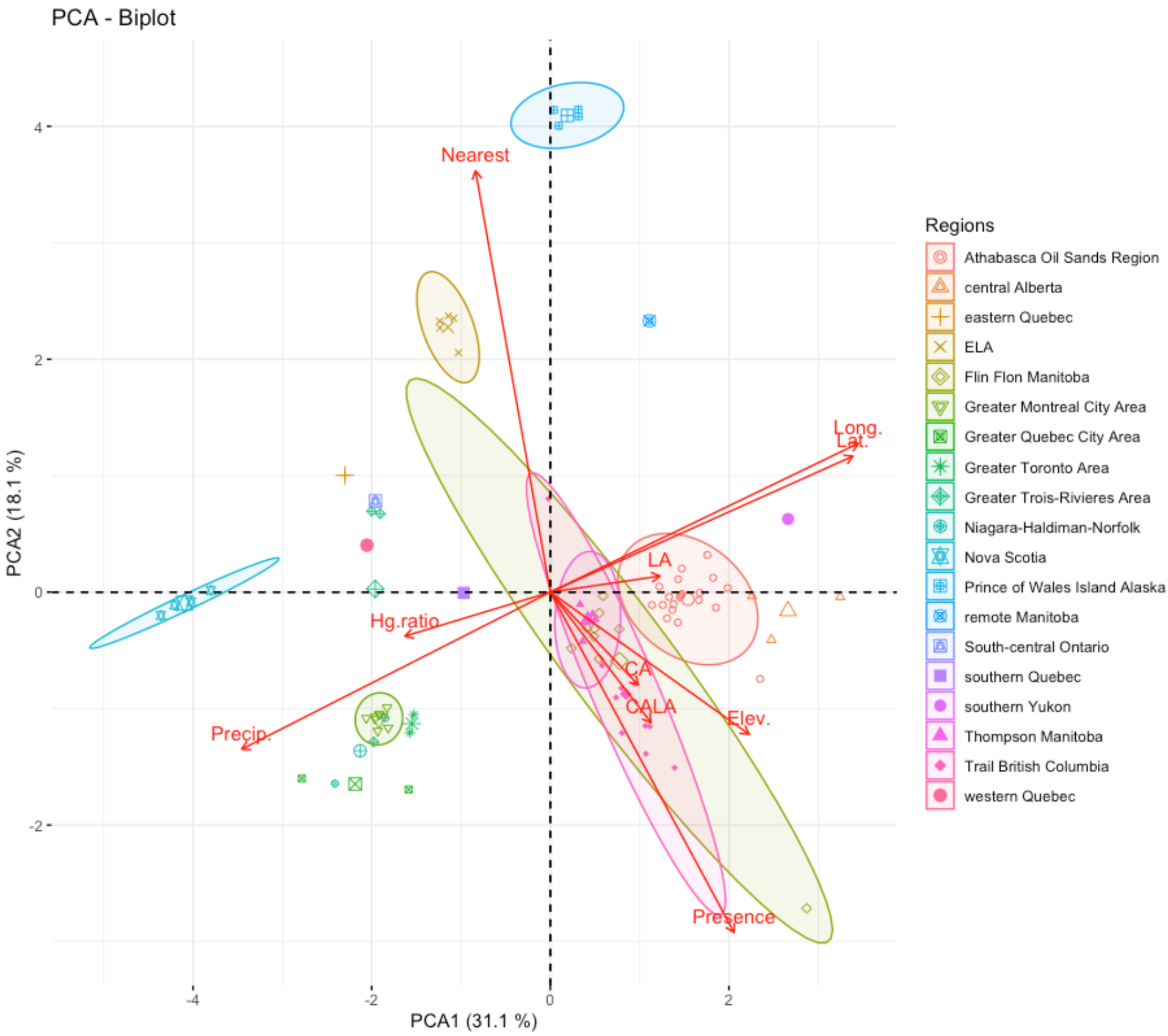

Figure S9. PCA of post-2005 $\mathrm{Hg}$ flux ratios and environmental factors; including post-2005 precipitation quantity, lake area (LA), catchment area (CA), lake area: catchment area (CA:LA), elevation, longitude, latitude, distance to nearest point source and presence/absence of point source. 

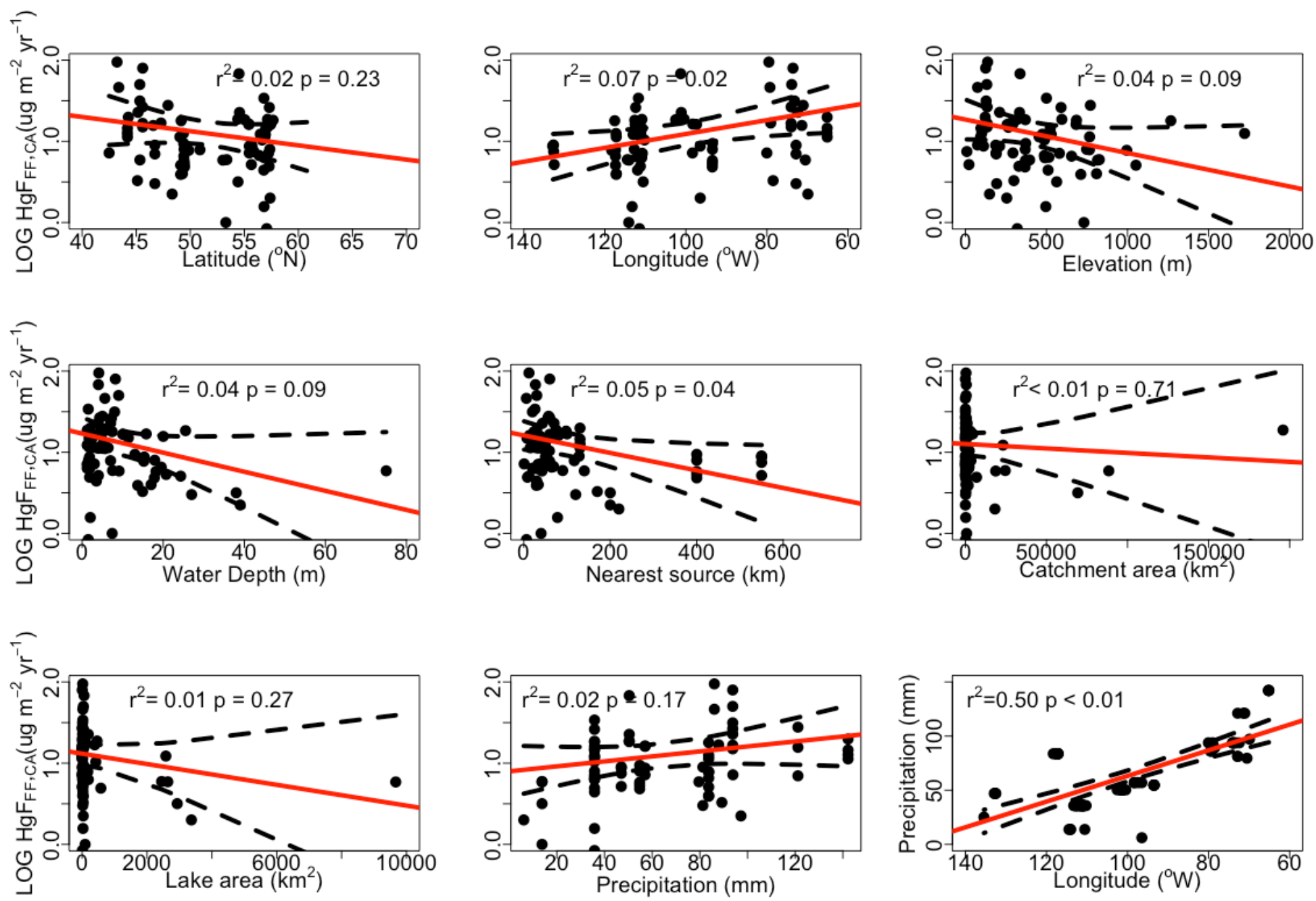

Figure S10. Pearson correlation with linear regression between logged average post-2005 anthropogenic $\mathrm{Hg}$ fluxes $\left(\mathrm{HgF}_{\mathrm{FF}, \mathrm{CA}}\right)$ and individual lake site $(\mathrm{n}=82)$ geographical location (latitude and longitude), elevation, water depth, distance to closest source, catchment area, lake area, average post-2005 precipitation quantity as well as between average post-2005 precipitation quantity and longitude. 

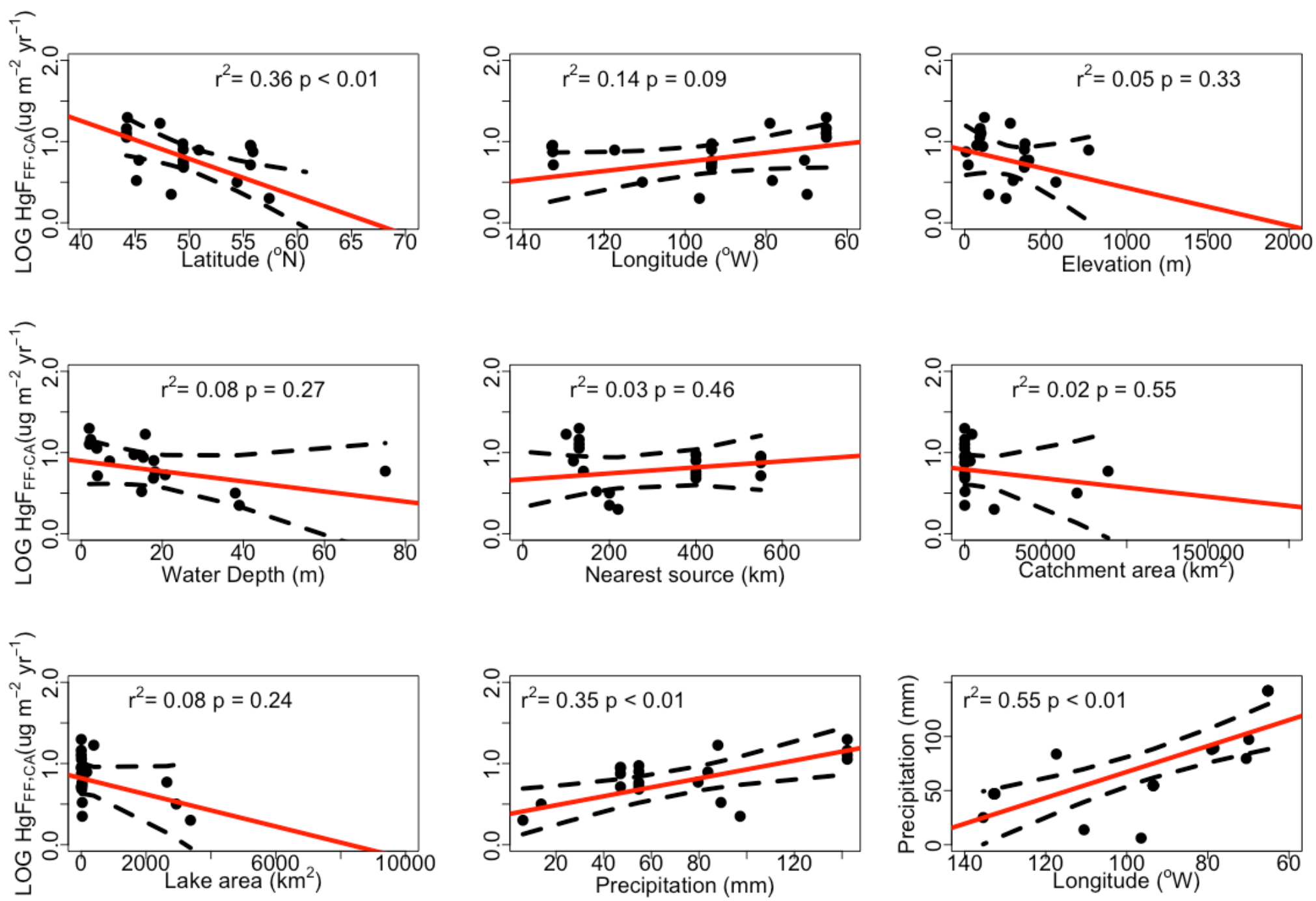

Figure S11. Pearson correlation with linear regression between logged average post-2005 anthropogenic $\mathrm{Hg}$ fluxes $\left(\mathrm{HgF}_{\mathrm{FF}, \mathrm{CA}}\right)$ and remote lake site $(\mathrm{n}=21)$ geographical location (latitude and longitude), elevation, water depth, distance to closest source, catchment area, lake area, average post-2005 precipitation quantity as well as between average post-2005 precipitation quantity and longitude. 


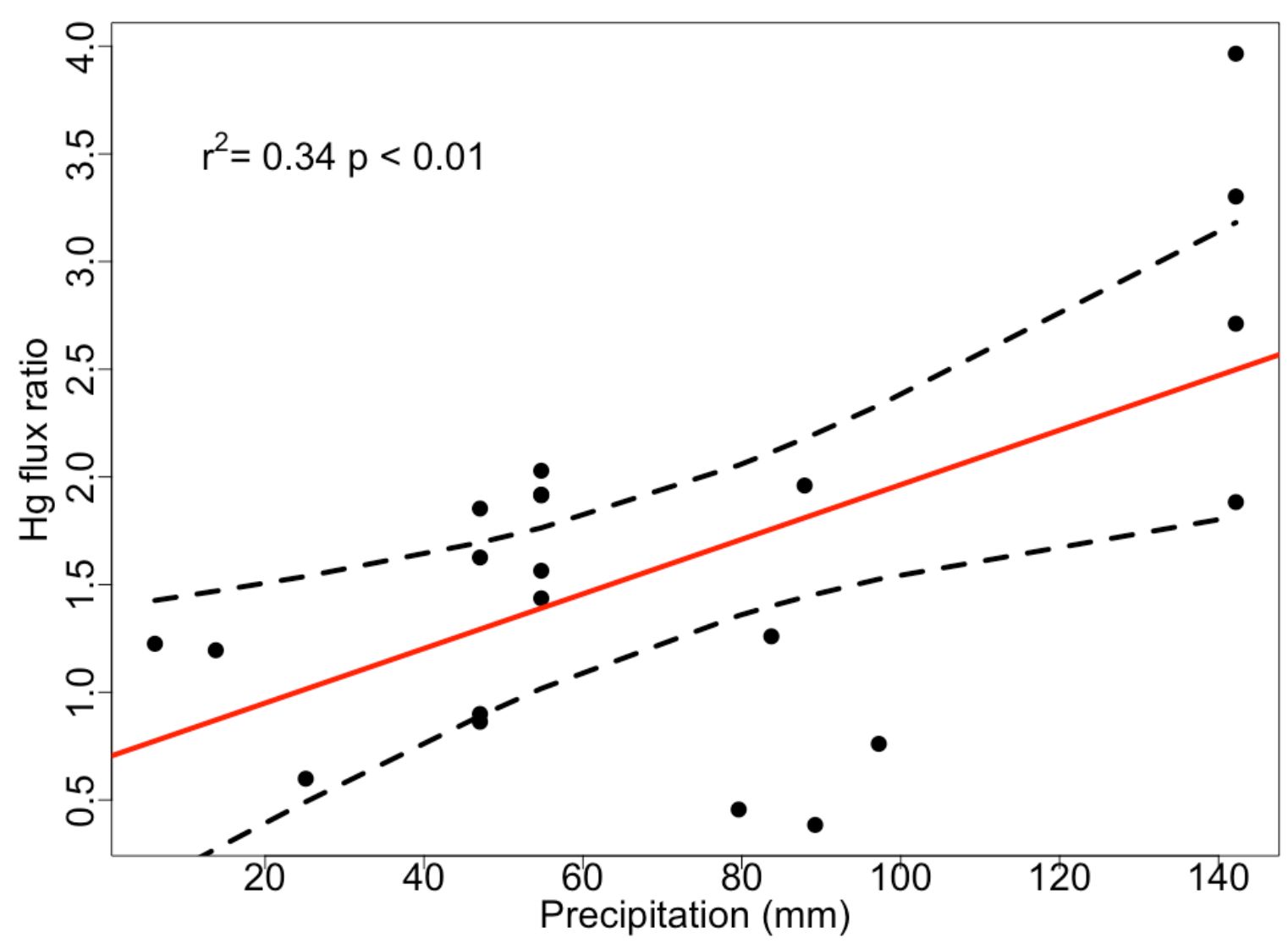

Figure S12. Pearson correlation between anthropogenic Hg flux ratio and post-2005 monthly mean precipitation quantity for the 21 remote sites sampled across Canada. 

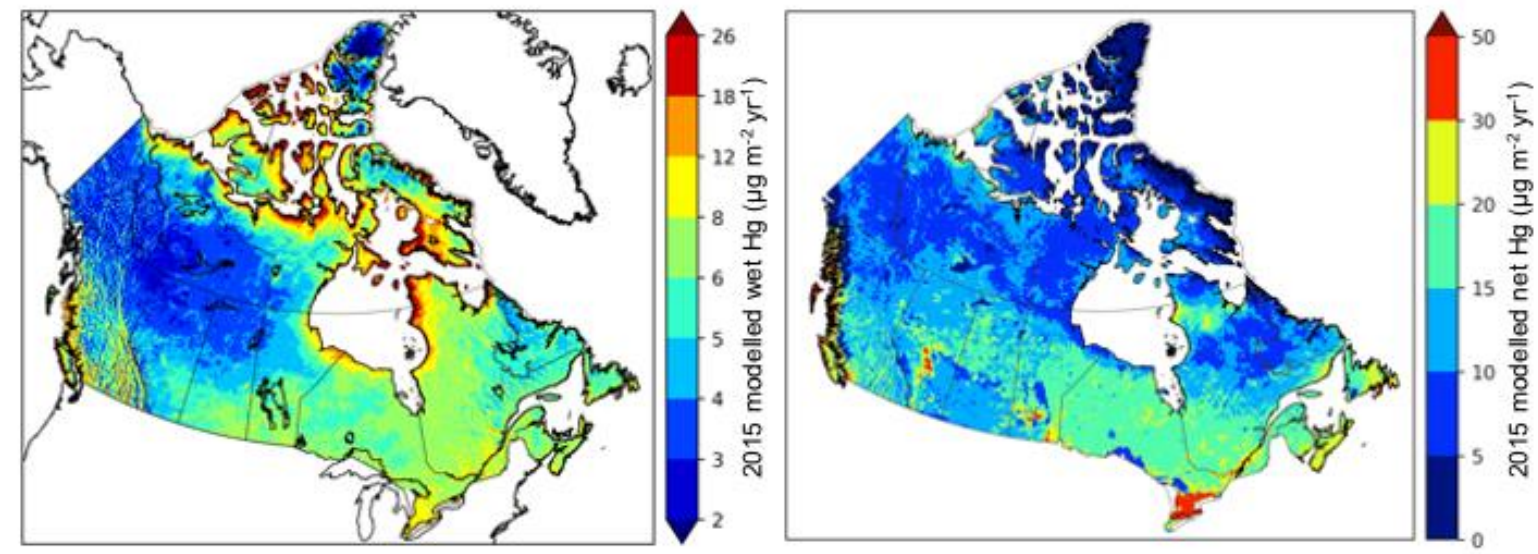

Figure S13. Modelled 2015 wet $\mathrm{Hg}$ deposition to lake surface and net Hg deposition to catchment across Canada obtained using GEM-MACH-Hg model. 
Table S1. Location of 82 lakes from 19 regions across Canada and Prince of Wales Island in southern Alaska sampled for sediment cores, year of core collection and their distance $(\mathrm{km})$ to nearest point source, and terrestrial ecozone. Regions located near industrial and/or urban point sources are denoted with bold text. For the central Alberta region lakes, both the distance to the Wabamun coal-fired power plant and [distance to Edmonton City] are shown. For southern Yukon, the lake site is considered remote due to the small size of the nearby city $(0.15 \%$ the size of Greater Toronto Area).

\begin{tabular}{|c|c|c|c|c|c|c|c|}
\hline Region & Lake site & Region type & $\begin{array}{c}\text { Core } \\
\text { collection }\end{array}$ & ${ }^{\circ} \mathbf{N}$ & ${ }^{0} \mathbf{W}$ & $\begin{array}{l}\text { Terrestrial } \\
\text { Ecozone }\end{array}$ & $\begin{array}{c}\text { Distance to } \\
\text { closest } \\
\text { point } \\
\text { source } \\
(\mathbf{k m}) \\
\end{array}$ \\
\hline southern Yukon & Scout-1 & Remote & 2013 & 60.79 & -135.43 & $\begin{array}{c}\text { Boreal } \\
\text { Cordillera }\end{array}$ & 18.0 \\
\hline \multirow{4}{*}{$\begin{array}{l}\text { Prince of Wales } \\
\text { Island, Alaska }\end{array}$} & Control & \multirow{4}{*}{ Remote } & \multirow{4}{*}{2012} & 55.69 & -132.87 & $\begin{array}{l}\text { Pacific } \\
\text { Maritime }\end{array}$ & 550.0 \\
\hline & Mountain Goat & & & 55.65 & -132.67 & $\begin{array}{c}\text { Pacific } \\
\text { Maritime }\end{array}$ & 550.0 \\
\hline & Trumpeter & & & 55.88 & -132.63 & $\begin{array}{c}\text { Pacific } \\
\text { Maritime }\end{array}$ & 550.0 \\
\hline & Cabin & & & 55.65 & -132.50 & $\begin{array}{c}\text { Pacific } \\
\text { Maritime }\end{array}$ & 550.0 \\
\hline \multirow{10}{*}{$\begin{array}{c}\text { Trail, British } \\
\text { Columbia }\end{array}$} & Wilgress & \multirow{3}{*}{ Industrial } & \multirow{10}{*}{2016} & 49.85 & -118.32 & $\begin{array}{l}\text { Montane } \\
\text { Cordillera }\end{array}$ & 59.7 \\
\hline & Nancy Greene & & & 49.15 & -117.57 & $\begin{array}{l}\text { Montane } \\
\text { Cordillera }\end{array}$ & 23.7 \\
\hline & $\begin{array}{l}\text { Upper Little } \\
\text { Slocan }\end{array}$ & & & 49.41 & -117.39 & $\begin{array}{l}\text { Montane } \\
\text { Cordillera }\end{array}$ & 64.2 \\
\hline & Summit & Remote & & 50.89 & -117.37 & $\begin{array}{l}\text { Montane } \\
\text { Cordillera }\end{array}$ & 116.8 \\
\hline & Champion & \multirow{6}{*}{ Industrial } & & 49.12 & -117.37 & $\begin{array}{l}\text { Montane } \\
\text { Cordillera }\end{array}$ & 12.1 \\
\hline & Bridal & & & 49.36 & -117.24 & $\begin{array}{l}\text { Montane } \\
\text { Cordillera }\end{array}$ & 49.7 \\
\hline & Erie & & & 49.11 & -117.21 & $\begin{array}{l}\text { Montane } \\
\text { Cordillera }\end{array}$ & 29.0 \\
\hline & Lomond & & & 49.04 & -117.19 & $\begin{array}{l}\text { Montane } \\
\text { Cordillera }\end{array}$ & 31.7 \\
\hline & Rosebud & & & 49.27 & -117.16 & $\begin{array}{l}\text { Montane } \\
\text { Cordillera }\end{array}$ & 33.4 \\
\hline & Cottonwood & & & 49.26 & -117.15 & $\begin{array}{c}\text { Montane } \\
\text { Cordillera } \\
\end{array}$ & 49.8 \\
\hline \multirow{4}{*}{ central Alberta } & Isle & \multirow{3}{*}{ Urban/industrial } & 2009 & 53.38 & -114.45 & Boreal Plains & $18.0[90]$ \\
\hline & Hasse & & 2012 & 53.29 & -114.10 & Boreal Plains & $20.0[40]$ \\
\hline & Pigeon & & \multirow{2}{*}{2009} & 53.02 & -114.04 & Boreal Plains & $63.1[42]$ \\
\hline & Wolf & Remote & & 54.41 & -110.55 & Boreal Plains & 252.5 [205] \\
\hline \multirow{8}{*}{$\begin{array}{l}\text { Athabasca Oil } \\
\text { Sands Region }\end{array}$} & 2014-Y & \multirow{8}{*}{ Industrial } & \multirow{8}{*}{2011} & 56.82 & -113.27 & Boreal Plains & 78.0 \\
\hline & 2014-D & & & 56.95 & -113.03 & Boreal Plains & 43.4 \\
\hline & 2014-X & & & 57.36 & -112.73 & Boreal Plains & 54.2 \\
\hline & $\begin{array}{l}\text { RAMP } 464 \\
\text { (L60) }\end{array}$ & & & 57.65 & -112.62 & Boreal Plains & 97.9 \\
\hline & 2014-Z & & & 57.44 & -112.53 & Boreal Plains & 80.5 \\
\hline & $\begin{array}{l}\text { RAMP } 175 \\
\text { (P13) }\end{array}$ & & & 57.31 & -112.40 & Boreal Plains & 55.5 \\
\hline & RAMP 225 & & & 56.80 & -111.92 & Boreal Plains & 29.8 \\
\hline & $\begin{array}{l}\text { RAMP } 223 \\
\text { (NW35) }\end{array}$ & & & 56.78 & -111.79 & Boreal Plains & 20.1 \\
\hline
\end{tabular}




\begin{tabular}{|c|c|c|c|c|c|c|c|}
\hline & $\begin{array}{l}\text { RAMP226 } \\
\text { (SW22) }\end{array}$ & & & 56.81 & -111.72 & Boreal Plains & 25.9 \\
\hline & NE20 & & & 57.13 & -111.56 & Boreal Plains & 7.5 \\
\hline & NE13 & & & 57.07 & -111.48 & Boreal Plains & 6.3 \\
\hline & $\begin{array}{l}\text { RAMP } 418 \\
\text { (Kearle) }\end{array}$ & & & 57.29 & -111.24 & Boreal Plains & 8.5 \\
\hline & Gregoire & & & 56.48 & -111.15 & Boreal Plains & 25.5 \\
\hline & SE22 & & & 56.90 & -111.14 & Boreal Plains & 15.0 \\
\hline & 2014-C & & & 56.39 & -110.95 & Boreal Plains & 68.9 \\
\hline & $\begin{array}{l}\text { RAMP } 268 \\
\text { (E15) }\end{array}$ & & & 56.89 & -110.90 & Boreal Plains & 28.8 \\
\hline & RAMP 185 & & & 57.15 & -110.86 & Boreal Plains & 29.8 \\
\hline & 2014-B & & & 56.98 & -110.81 & Boreal Plains & 34.1 \\
\hline & RAMP 209 (P7) & & & 57.23 & -110.75 & Boreal Plains & 33.5 \\
\hline & RAMP 271 & & & 56.64 & -110.22 & Boreal Plains & 59.3 \\
\hline \multirow{9}{*}{$\begin{array}{l}\text { Flin Flon, } \\
\text { Manitoba }\end{array}$} & Mclurg & \multirow{9}{*}{ Industrial } & \multirow{9}{*}{2009} & 55.04 & -102.53 & Boreal shield & 75.0 \\
\hline & Hamel & & & 54.49 & -101.58 & Boreal shield & 4.5 \\
\hline & Meridian & & & 54.44 & -101.58 & Boreal shield & 4.5 \\
\hline & Douglas & & & 54.44 & -101.56 & Boreal shield & 5.0 \\
\hline & Phantom & & & 54.44 & -101.52 & Boreal shield & 5.0 \\
\hline & Cleaver & & & 54.42 & -101.33 & Boreal shield & 23.0 \\
\hline & Naosap Mud & & & 54.51 & -101.29 & Boreal shield & 27.0 \\
\hline & Nekik & & & 54.55 & -101.15 & Boreal shield & 43.0 \\
\hline & Loucks & & & 54.40 & -100.44 & Boreal shield & 75.0 \\
\hline \multirow{4}{*}{$\begin{array}{l}\text { Thompson, } \\
\text { Manitoba }\end{array}$} & TM-9A & \multirow{4}{*}{ Industrial } & \multirow{4}{*}{2014} & 55.45 & -98.38 & Boreal shield & 41.0 \\
\hline & TM-8A & & & 55.43 & -98.30 & Boreal shield & 70.0 \\
\hline & TM-1A & & & 55.42 & -97.39 & Boreal shield & 13.0 \\
\hline & TM-3A & & & 55.46 & -96.48 & Boreal shield & 50.0 \\
\hline $\begin{array}{c}\text { remote } \\
\text { Manitoba }\end{array}$ & T1111 & Remote & 2011 & 57.39 & -96.47 & Boreal shield & 220.0 \\
\hline \multirow{5}{*}{$\begin{array}{c}\text { Experimental } \\
\text { Lakes Area, } \\
\text { Ontario }\end{array}$} & L442 & \multirow{5}{*}{ Remote } & \multirow{5}{*}{2010} & 49.46 & -93.49 & Boreal shield & 400.0 \\
\hline & L373 & & & 49.45 & -93.48 & Boreal shield & 400.0 \\
\hline & L378 & & & 49.42 & -93.46 & Boreal shield & 400.0 \\
\hline & L337 & & & 49.43 & -93.46 & Boreal shield & 400.0 \\
\hline & L240 & & & 49.39 & -93.43 & Boreal shield & 400.0 \\
\hline \multirow{2}{*}{$\begin{array}{l}\text { Niagara- } \\
\text { Haldimand- } \\
\text { Norfolk, } \\
\text { Ontario }\end{array}$} & Nanticoke & \multirow[b]{2}{*}{ Industrial } & \multirow[b]{2}{*}{2016} & 42.49 & -80.04 & $\begin{array}{l}\text { Mixedwood } \\
\text { Plains }\end{array}$ & 0.8 \\
\hline & $\begin{array}{l}\text { Windmill Point } \\
\text { Pond }\end{array}$ & & & 42.53 & -79.00 & $\begin{array}{l}\text { Mixedwood } \\
\text { Plains }\end{array}$ & 75.0 \\
\hline \multirow{2}{*}{$\begin{array}{c}\text { Greater } \\
\text { Toronto Area, } \\
\text { Ontario }\end{array}$} & Medad & \multirow[b]{2}{*}{ Urban } & 2016 & 43.22 & -79.53 & $\begin{array}{l}\text { Mixedwood } \\
\text { Plains }\end{array}$ & 12.0 \\
\hline & Grenadier & & 2017 & 43.38 & -79.28 & $\begin{array}{l}\text { Mixedwood } \\
\text { Plains }\end{array}$ & 6.0 \\
\hline $\begin{array}{l}\text { remote south- } \\
\text { central Ontario }\end{array}$ & Plastic & Remote & 2016 & 45.11 & -78.49 & $\begin{array}{l}\text { Mixedwood } \\
\text { Plains }\end{array}$ & 170.0 \\
\hline
\end{tabular}




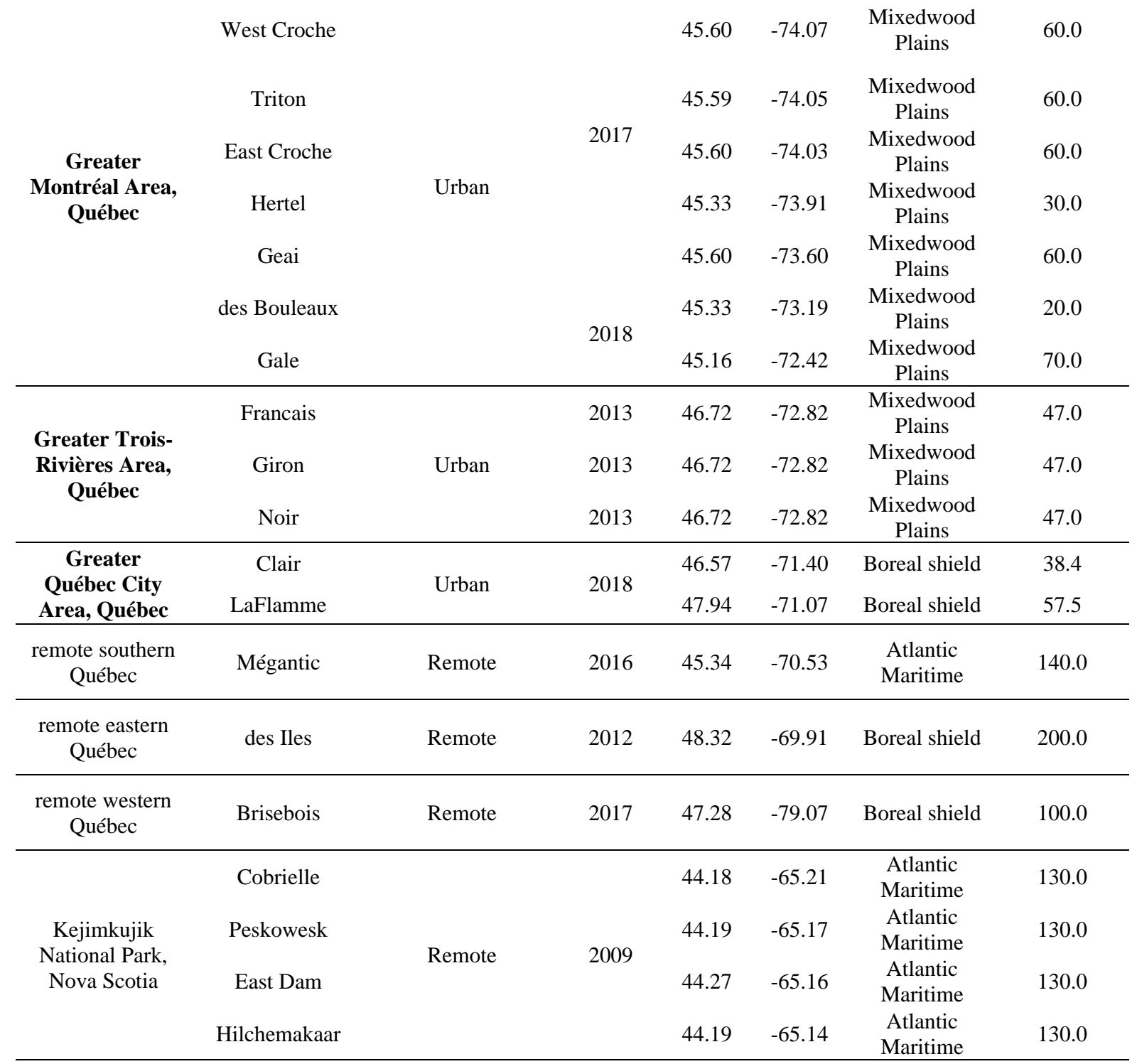

Table S2. Water depth, elevation, lake area, catchment area and catchment area: lake area ratios for all lake sites cored across Canada and Prince of Wales Island, Alaska. Regions located near industrial and/or urban point sources are denoted with bold text.

\begin{tabular}{|c|c|c|c|c|c|c|c|c|}
\hline Region & Lake site & $\begin{array}{l}\text { Region } \\
\text { type }\end{array}$ & ${ }^{\mathbf{o}} \mathbf{N}$ & ${ }^{\circ} \mathbf{W}$ & $\begin{array}{c}\text { Water } \\
\text { depth } \\
(\mathbf{m})\end{array}$ & $\begin{array}{l}\text { Elevation } \\
\quad(\mathbf{m})\end{array}$ & $\begin{array}{l}\text { Lake area } \\
\text { (ha) }\end{array}$ & $\begin{array}{l}\text { Catchment } \\
\text { area (ha) }\end{array}$ \\
\hline southern Yukon & Scout-1 & Remote & 60.79 & -135.43 & 12.8 & 323 & 22.6 & 391.3 \\
\hline \multirow{4}{*}{$\begin{array}{c}\text { Prince of Wales Island, } \\
\text { Alaska }\end{array}$} & Control & \multirow{4}{*}{ Remote } & 55.69 & -132.87 & 15.3 & 112 & 7.6 & 102.5 \\
\hline & Mountain Goat & & 55.65 & -132.67 & $(-)$ & 75 & 2.8 & 17.6 \\
\hline & Trumpeter & & 55.88 & -132.63 & $(-)$ & 9 & 6.9 & 35.7 \\
\hline & Cabin & & 55.65 & -132.50 & 4.0 & 22 & 0.1 & 3.2 \\
\hline \multirow{4}{*}{ Trail, British Columbia } & Wilgress & & 49.85 & -118.32 & 15.2 & 995 & 17.8 & 205.4 \\
\hline & Nancy Greene & Industrial & 49.15 & -117.57 & 7.4 & 1,267 & 34.0 & $1,481.0$ \\
\hline & Upper Little Slocan & & 49.41 & -117.39 & 19.5 & 655 & 74.5 & $2,287.0$ \\
\hline & Summit & Remote & 50.89 & -117.37 & 7.0 & 765 & 171.9 & $3,395.5$ \\
\hline
\end{tabular}




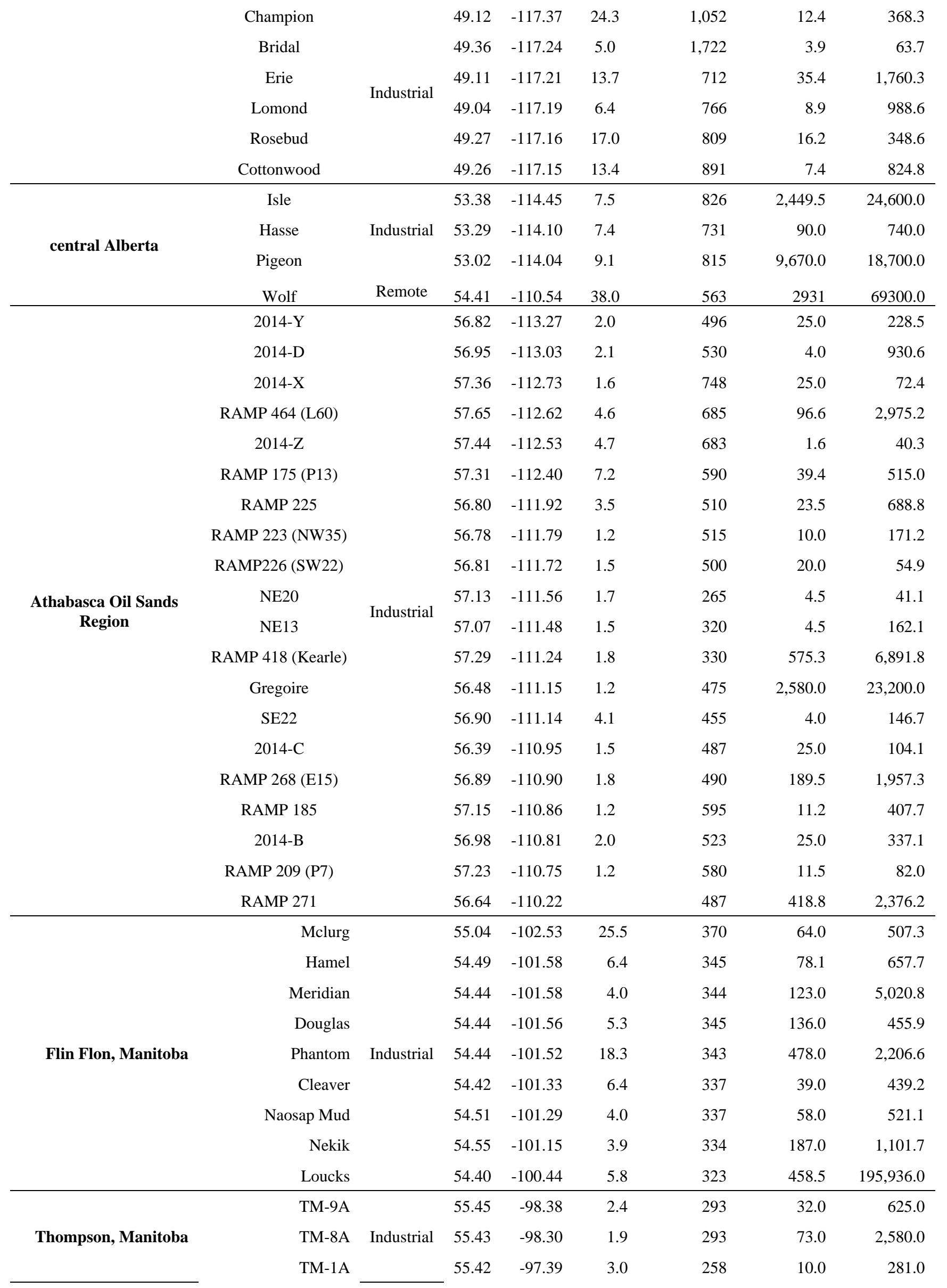




\begin{tabular}{|c|c|c|c|c|c|c|c|c|}
\hline & TM-3A & & 55.46 & -96.48 & 1.8 & 211 & 73.0 & 506.0 \\
\hline remote Manitoba & $\mathrm{T} 1111$ & Remote & 57.39 & -96.47 & $(-)$ & 255 & $3,368.3$ & $18,239.2$ \\
\hline \multirow{5}{*}{$\begin{array}{r}\text { Experimental Lakes } \\
\text { Area, Ontario }\end{array}$} & L442 & \multirow{5}{*}{ Remote } & 49.46 & -93.49 & 17.8 & 368 & 16.0 & 168.4 \\
\hline & L373 & & 49.45 & -93.48 & 20.7 & 368 & 27.3 & 53.9 \\
\hline & L378 & & 49.42 & -93.46 & 18.2 & 370 & 24.3 & 110.1 \\
\hline & L337 & & 49.43 & -93.46 & 17.9 & 369 & 26.9 & $2,002.8$ \\
\hline & L240 & & 49.39 & -93.43 & 13.0 & 372 & 44.1 & 688.9 \\
\hline \multirow{2}{*}{$\begin{array}{r}\text { Niagara-Haldimand- } \\
\text { Norfolk, Ontario }\end{array}$} & Nanticoke & \multirow[t]{2}{*}{ Industrial } & 42.49 & -80.04 & 3.0 & 189 & 4.9 & 156.8 \\
\hline & Windmill Point Pond & & 42.53 & -79.00 & 6.1 & 193 & 6.1 & 156.5 \\
\hline \multirow{2}{*}{$\begin{array}{r}\text { Greater Toronto Area, } \\
\text { Ontario }\end{array}$} & Medad & \multirow{2}{*}{ Urban } & 43.22 & -79.53 & 4.1 & 137 & 16.0 & 377.6 \\
\hline & Grenadier & & 43.38 & -79.28 & 5.6 & 75 & 20.6 & 24.5 \\
\hline $\begin{array}{r}\text { remote South-central } \\
\text { Ontario }\end{array}$ & Plastic & Remote & 45.11 & -78.49 & 14.9 & 299 & 32.0 & 254.2 \\
\hline \multirow{7}{*}{$\begin{array}{r}\text { Greater Montréal } \\
\text { Area, Québec }\end{array}$} & West Croche & \multirow{7}{*}{ Urban } & 45.60 & -74.07 & 10.0 & 134 & 10.9 & 103.9 \\
\hline & Triton & & 45.59 & -74.05 & 4.3 & 134 & 1.8 & 25.7 \\
\hline & East Croche & & 45.60 & -74.03 & 11.4 & 134 & 6.1 & 103.9 \\
\hline & Hertel & & 45.33 & -73.91 & 9.0 & 132 & 36.6 & 457.6 \\
\hline & Geai & & 45.60 & -73.60 & 8.2 & 125 & 1.1 & 24.6 \\
\hline & des Bouleaux & & 45.33 & -73.19 & 8.0 & 128 & 12.9 & 59.7 \\
\hline & Gale & & 45.16 & -72.42 & 7.0 & 211 & 11.7 & 71.6 \\
\hline \multirow{3}{*}{$\begin{array}{c}\text { Greater Trois-Rivières } \\
\text { Area, Québec }\end{array}$} & Francais & \multirow{3}{*}{ Urban } & 46.72 & -72.82 & 27.0 & 193 & 17.0 & 181.2 \\
\hline & Giron & & 46.72 & -72.82 & $(-)$ & 187 & 35.4 & 334.2 \\
\hline & Noir & & 46.72 & -72.82 & $(-)$ & 190 & 8.6 & 45.6 \\
\hline \multirow{2}{*}{$\begin{array}{c}\text { Greater Québec City } \\
\text { Area, Québec }\end{array}$} & Clair & \multirow{2}{*}{ Urban } & 46.57 & -71.40 & 20.0 & 274 & 44.4 & 196.6 \\
\hline & Laflamme & & 47.94 & -71.07 & 5.0 & 771 & 6.4 & 88.4 \\
\hline Remote southern Québec & Mégantic & Remote & 45.34 & -70.53 & 75.0 & 396 & $2,640.0$ & $88,446.7$ \\
\hline Remote eastern Québec & des Iles & Remote & 48.32 & -69.91 & 39.0 & 149 & 32.4 & 40.3 \\
\hline Remote western Québec & Brisbois & Remote & 47.28 & -79.07 & 15.8 & 282 & 386.5 & 4527.0 \\
\hline \multirow{4}{*}{$\begin{array}{r}\text { Kejimkujik National } \\
\text { Park, Nova Scotia }\end{array}$} & Cobrielle & \multirow{4}{*}{ Remote } & 44.18 & -65.21 & 2.0 & 101 & 1.0 & 3.8 \\
\hline & Peskowesk & & 44.19 & -65.17 & 2.3 & 96 & 0.5 & 85.0 \\
\hline & East Dam & & 44.27 & -65.16 & 2.0 & 122 & 1.4 & 11.5 \\
\hline & Hilchemakaar & & 44.19 & -65.14 & 3.9 & 94 & 7.4 & 2.0 \\
\hline
\end{tabular}


Table S3. Baseline period applied to each of the 82 lake sediment cores to calculate anthropogenic Hg fluxes. 2 of the 15 Québec sediment cores did not extend back to background levels (pre-1850) and average baseline $\mathrm{Hg}$ concentration values from lake sediment cores within the same region were thus used to establish anthropogenic $\mathrm{Hg}$ fluxes. Similarly, 4 of the 5 southern Ontario cores did not extend back to baseline levels (pre-1850) and an average baseline value for $\mathrm{Hg}$ concentrations from a Lake Ontario sediment core (Fig. S4) was used to establish anthropogenic Hg fluxes.

\begin{tabular}{|c|c|c|c|}
\hline Region & Lake site & $\begin{array}{c}\text { Baseline } \\
\text { applied }\end{array}$ & Rationale \\
\hline southern Yukon & Scout-1 & \multirow{5}{*}{ pre-1880 } & \multirow{5}{*}{ Gold rush in late $1800 \mathrm{~s}$} \\
\hline \multirow{4}{*}{$\begin{array}{r}\text { Prince of Wales Island, } \\
\text { Alaska }\end{array}$} & Control & & \\
\hline & Mountain Goat & & \\
\hline & Trumpeter & & \\
\hline & Cabin & & \\
\hline \multirow{10}{*}{ Trail, British Columbia } & Wilgress & \multirow{10}{*}{ pre-1840 } & \multirow{10}{*}{$\begin{array}{l}\text { Mining and smelting operations began } \\
\text { in the mid-late } 1800 \mathrm{~s}\end{array}$} \\
\hline & Nancy Greene & & \\
\hline & Upper Little Slocan & & \\
\hline & Summit & & \\
\hline & Champion & & \\
\hline & Bridal & & \\
\hline & Erie & & \\
\hline & Lomond & & \\
\hline & Rosebud & & \\
\hline & Cottonwood & & \\
\hline \multirow{4}{*}{ central Alberta } & Isle & \multirow{4}{*}{ pre-1915 } & \multirow{4}{*}{ Prior to industrialisation in the region } \\
\hline & Hasse & & \\
\hline & Pigeon & & \\
\hline & Wolf & & \\
\hline \multirow{13}{*}{ Athabasca Oil Sands Region } & $2014-\mathrm{Y}$ & \multirow{13}{*}{ pre-1850 } & \multirow{13}{*}{ Prior to industrialisation in the region } \\
\hline & 2014-D & & \\
\hline & 2014-X & & \\
\hline & RAMP 464 (L60) & & \\
\hline & 2014-Z & & \\
\hline & RAMP 175 (P13) & & \\
\hline & RAMP 225 & & \\
\hline & RAMP 223 (NW35) & & \\
\hline & RAMP226 (SW22) & & \\
\hline & NE20 & & \\
\hline & NE13 & & \\
\hline & RAMP 418 (Kearle) & & \\
\hline & Gregoire & & \\
\hline
\end{tabular}


RAMP 268 (E15)

RAMP 185

2014-B

RAMP 209 (P7)

RAMP 271

\begin{tabular}{|c|c|c|c|}
\hline \multirow{9}{*}{ Flin Flon, Manitoba } & Mclurg & \multirow{14}{*}{ pre-1915 } & \multirow{14}{*}{$\begin{array}{c}\text { Early mine development began between } \\
1917 \text { and } 1920 \text { in the Flin Flon } \\
\text { Manitoba region }\end{array}$} \\
\hline & Hamel & & \\
\hline & Meridian & & \\
\hline & Douglas & & \\
\hline & Phantom & & \\
\hline & Cleaver & & \\
\hline & Naosap Mud & & \\
\hline & Nekik & & \\
\hline & Loucks & & \\
\hline \multirow{4}{*}{ Thompson, Manitoba } & TM-9A & & \\
\hline & TM-8A & & \\
\hline & TM-1A & & \\
\hline & TM-3A & & \\
\hline remote Manitoba & T1111 & & \\
\hline \multirow{5}{*}{$\begin{array}{r}\text { Experimental Lakes Area, } \\
\text { Ontario }\end{array}$} & L442 & \multirow{5}{*}{ pre-1860 } & \multirow{5}{*}{$\begin{array}{l}\text { Prior to onset of smelter activities in } \\
\text { Michigan's Northern Peninsula }\end{array}$} \\
\hline & L373 & & \\
\hline & L378 & & \\
\hline & L337 & & \\
\hline & L240 & & \\
\hline \multirow{2}{*}{$\begin{array}{r}\text { Niagara-Haldimand-Norfolk, } \\
\text { Ontario }\end{array}$} & Nanticoke & \multirow{15}{*}{ pre-1850 } & \multirow{15}{*}{ Prior to onset of urban related emissions } \\
\hline & Windmill Point Pond & & \\
\hline \multirow{2}{*}{$\begin{array}{r}\text { Greater Toronto Area, } \\
\text { Ontario }\end{array}$} & Medad & & \\
\hline & Grenadier & & \\
\hline remote southern Ontario & Plastic & & \\
\hline \multirow{7}{*}{$\begin{array}{r}\text { Greater Montréal } \\
\text { Area, Québec }\end{array}$} & West Croche & & \\
\hline & Triton & & \\
\hline & East Croche & & \\
\hline & Hertel & & \\
\hline & Geai & & \\
\hline & des Bouleaux & & \\
\hline & Gale & & \\
\hline \multirow{3}{*}{$\begin{array}{l}\text { Greater Trois-Rivières } \\
\text { Area, Québec }\end{array}$} & Francais & & \\
\hline & Giron & & \\
\hline & Noir & & \\
\hline
\end{tabular}




\begin{tabular}{|c|c|c|c|}
\hline $\begin{array}{l}\text { Greater Québec City } \\
\text { Area, Québec }\end{array}$ & $\begin{array}{r}\text { Clair } \\
\text { Laflamme }\end{array}$ & & \\
\hline Remote southern Québec & Mégantic & & \\
\hline Remote eastern Québec & des Iles & & \\
\hline Remote western Québec & Brisebois & & \\
\hline \multirow[b]{2}{*}{$\begin{array}{r}\text { Kejimkujik National Park, } \\
\text { Nova Scotia }\end{array}$} & Cobrielle & & \\
\hline & $\begin{array}{r}\text { Peskowesk } \\
\text { East Dam } \\
\text { Hilchemakkar }\end{array}$ & pre-1800 & $\begin{array}{c}\text { Prior to east coast North American } \\
\text { industrialisation and urban related } \\
\text { emissions }\end{array}$ \\
\hline
\end{tabular}

Table S4. ${ }^{210} \mathrm{~Pb}$ dating laboratories for the 82 lake sediment core chronologies: ECCC Canada Centre for Inland Waters (CCIW) Burlington ON, Institut National de la Recherche Scientifique (INRS) Québec and the Flett Research Ltd in Winnipeg MB.

\begin{tabular}{|c|c|c|}
\hline Region & Lake site & ${ }^{210} \mathrm{~Pb}$ dating lab \\
\hline southern Yukon & Scout-1 & INRS Québec \\
\hline \multirow{4}{*}{ Prince of Wales Island, Alaska } & Control & \multirow{4}{*}{ INRS Québec } \\
\hline & Mountain Goat & \\
\hline & Trumpeter & \\
\hline & Cabin & \\
\hline \multirow{10}{*}{ Trail, British Columbia } & Wilgress & \multirow{10}{*}{ CCIW } \\
\hline & Nancy Greene & \\
\hline & Upper Little Slocan & \\
\hline & Summit & \\
\hline & Champion & \\
\hline & Bridal & \\
\hline & Erie & \\
\hline & Lomond & \\
\hline & Rosebud & \\
\hline & Cottonwood & \\
\hline \multirow{4}{*}{ central Alberta } & Isle & Flett Research Ltd. \\
\hline & Hasse & INRS Québec \\
\hline & Pigeon & \multirow{2}{*}{ Flett Research Ltd. } \\
\hline & Wolf & \\
\hline \multirow{7}{*}{ Athabasca Oil Sands Region } & 2014-Y & \multirow{7}{*}{ Flett Research Ltd. } \\
\hline & 2014-D & \\
\hline & $2014-X$ & \\
\hline & RAMP 464 (L60) & \\
\hline & $2014-Z$ & \\
\hline & RAMP 175 (P13) & \\
\hline & RAMP 225 & \\
\hline
\end{tabular}


RAMP 223 (NW35)

RAMP226 (SW22)

NE20

NE13

RAMP 418 (Kearle)

Gregoire

SE22

2014-C

RAMP 268 (E15)

RAMP 185

2014-B

RAMP 209 (P7)

RAMP 271

\begin{tabular}{|c|c|c|}
\hline \multirow{9}{*}{ Flin Flon, Manitoba } & Mclurg & \multirow{9}{*}{ CCIW } \\
\hline & Hamel & \\
\hline & Meridian & \\
\hline & Douglas & \\
\hline & Phantom & \\
\hline & Cleaver & \\
\hline & Naosap Mud & \\
\hline & Nekik & \\
\hline & Loucks & \\
\hline \multirow{4}{*}{ Thompson, Manitoba } & TM-9A & \multirow{4}{*}{ CCIW } \\
\hline & TM-8A & \\
\hline & TM-1A & \\
\hline & TM-3A & \\
\hline remote Manitoba & T1111 & Flett Research Ltd. \\
\hline \multirow{5}{*}{ Experimental Lakes Area, Ontario } & L442 & \multirow{5}{*}{ CCIW } \\
\hline & L373 & \\
\hline & L378 & \\
\hline & L337 & \\
\hline & L240 & \\
\hline \multirow{2}{*}{ Niagara-Haldimand-Norfolk, Ontario } & Nanticoke & \multirow{2}{*}{ CCIW } \\
\hline & Windmill Point Pond & \\
\hline \multirow{2}{*}{ Greater Toronto Area, Ontario } & Medad & \multirow{2}{*}{ CCIW } \\
\hline & Grenadier & \\
\hline remote South-central Ontario & Plastic & CCIW \\
\hline \multirow{4}{*}{ Greater Montréal Area, Québec } & West Croche & \multirow{4}{*}{ CCIW } \\
\hline & Triton & \\
\hline & East Croche & \\
\hline & Hertel & \\
\hline
\end{tabular}




\begin{tabular}{ccc} 
& Gale & \\
Greater Trois-Rivières Area, Québec & Francais & INRS Québec \\
\hline Greater Québec City area, Québec & Giron & Noir \\
\hline Remote southern Québec & Clair & CCIW \\
\hline Remote eastern Québec & Laflamme & CCIW \\
\hline Remote western Québec & Mégantic & INRS Québec \\
\hline Kejimkujik National Park, Nova Scotia & des Iles & CCIW \\
& Brisebois & \\
\hline & Cobrielle & CCIW \\
\hline
\end{tabular}

Table S5. Average post-1990 sedimentation rates for each lake sediment core collected across Canada and Alaska.

\begin{tabular}{|c|c|c|c|c|c|}
\hline Region & Lake site & $\begin{array}{l}\text { Latitude } \\
\quad\left({ }^{\circ} \mathbf{N}\right)\end{array}$ & $\begin{array}{l}\text { Longitude } \\
\quad\left({ }^{\circ} \mathbf{W}\right)\end{array}$ & $\begin{array}{l}\text { post-1990 Avg. } \\
\text { sedimentation } \\
\text { rates }\left(\mathrm{g} \mathrm{cm}^{-2} \mathrm{yr}^{-1}\right)\end{array}$ & $\begin{array}{l}\text { post-1990 stdev. } \\
\text { sedimentation } \\
\text { rates }\left(\mathrm{g} \mathrm{cm}^{-2} \mathrm{yr}^{-1}\right)\end{array}$ \\
\hline Southern Yukon & Scout-1 & 60.79 & -135.43 & 0.012 & 0.002 \\
\hline \multirow[t]{4}{*}{ Prince of Wales Island } & Control & 55.69 & -132.87 & 0.011 & 0.001 \\
\hline & Mountain Goat & 55.65 & -132.67 & 0.049 & 0.026 \\
\hline & Trumpeter & 55.88 & -132.63 & 0.019 & 0.002 \\
\hline & Cabin & 55.65 & -132.50 & 0.003 & 0.001 \\
\hline \multirow[t]{10}{*}{ Trail British Columbia } & Wilgress & 49.85 & -118.32 & 0.002 & 0.001 \\
\hline & Nancy Greene & 49.15 & -117.57 & 0.020 & 0.002 \\
\hline & Upper Little Slocan & 49.41 & -117.39 & 0.127 & 0.018 \\
\hline & Summit & 50.89 & -117.37 & 0.009 & 0.004 \\
\hline & Champion & 49.12 & -117.37 & 0.006 & 0.001 \\
\hline & Bridal & 49.36 & -117.24 & 0.016 & 0.007 \\
\hline & Erie & 49.11 & -117.21 & 0.010 & 0.004 \\
\hline & Lomond & 49.04 & -117.19 & 0.029 & 0.003 \\
\hline & Rosebud & 49.27 & -117.16 & 0.001 & 0.001 \\
\hline & Cottonwood & 49.26 & -117.15 & 0.007 & 0.003 \\
\hline \multirow[t]{3}{*}{ central Alberta } & Isle & 53.38 & -114.45 & 0.024 & 0.006 \\
\hline & Hasse & 53.29 & -114.10 & 0.041 & 0.011 \\
\hline & Pigeon & 53.02 & -114.04 & 0.027 & 0.003 \\
\hline \multirow[t]{2}{*}{ Athabasca Oil Sands Region } & 2014-Y & 56.82 & -113.27 & 0.020 & 0.002 \\
\hline & 2014-D & 56.95 & -113.03 & 0.028 & 0.002 \\
\hline
\end{tabular}




\begin{tabular}{|c|c|c|c|c|c|}
\hline & 2014-X & 57.36 & -112.73 & 0.026 & 0.010 \\
\hline & RAMP464 & 57.65 & -112.62 & 0.027 & 0.002 \\
\hline & 2014-Z & 57.44 & -112.53 & 0.022 & 0.003 \\
\hline & RAMP175 & 57.31 & -112.40 & 0.054 & 0.028 \\
\hline & RAMP225 & 56.80 & -111.92 & 0.011 & 0.002 \\
\hline & RAMP223 & 56.78 & -111.79 & 0.010 & 0.002 \\
\hline & RAMP226 & 56.81 & -111.72 & 0.036 & 0.008 \\
\hline & NE20 & 57.13 & -111.56 & 0.029 & 0.006 \\
\hline & NE13 & 57.07 & -111.48 & 0.025 & 0.004 \\
\hline & RAMP418 & 57.29 & -111.24 & 0.015 & 0.004 \\
\hline & Gregoire & 56.48 & -111.15 & 0.066 & 0.005 \\
\hline & SE22 & 56.90 & -111.14 & 0.018 & 0.004 \\
\hline & 2014-C & 56.39 & -110.95 & 0.012 & 0.001 \\
\hline & RAMP268 & 56.89 & -110.90 & 0.012 & 0.003 \\
\hline & RAMP185 & 57.15 & -110.86 & 0.021 & 0.001 \\
\hline & 2014-B & 56.98 & -110.81 & 0.016 & 0.002 \\
\hline & RAMP209 & 57.23 & -110.75 & 0.014 & 0.001 \\
\hline central Alberta & Wolf & 54.41 & -110.55 & 0.020 & 0.002 \\
\hline Athabasca Oil Sands Region & RAMP271 & 56.64 & -110.22 & 0.010 & 0.000 \\
\hline Flin Flon & Mclurg & 55.04 & -102.53 & 0.019 & 0.006 \\
\hline & Hamel & 54.49 & -101.58 & 0.009 & 0.002 \\
\hline & Meridian & 54.44 & -101.58 & 0.024 & 0.003 \\
\hline & Douglas & 54.44 & -101.56 & 0.014 & 0.002 \\
\hline & Phantom & 54.44 & -101.52 & 0.023 & 0.001 \\
\hline & Cleaver & 54.42 & -101.33 & 0.022 & 0.004 \\
\hline & Nao & 54.51 & -101.29 & 0.011 & 0.002 \\
\hline & Nekik & 54.55 & -101.15 & 0.016 & 0.002 \\
\hline & Louck & 54.40 & -100.44 & 0.022 & 0.003 \\
\hline Thompson & TM-9 & 55.45 & -98.38 & 0.012 & 0.001 \\
\hline & TM-8 & 55.43 & -98.30 & 0.013 & 0.001 \\
\hline & TM-1 & 55.42 & -97.39 & 0.009 & 0.002 \\
\hline & TM-3 & 55.46 & -96.48 & 0.011 & 0.001 \\
\hline remote Manitoba & $\mathrm{T} 1111$ & 57.39 & -96.47 & 0.029 & 0.004 \\
\hline ELA & L442 & 49.46 & -93.49 & 0.010 & 0.001 \\
\hline & L373 & 49.45 & -93.48 & 0.007 & 0.001 \\
\hline & L378 & 49.42 & -93.46 & 0.016 & 0.002 \\
\hline & L337 & 49.43 & -93.46 & 0.009 & 0.001 \\
\hline & L240 & 49.39 & -93.43 & 0.024 & 0.002 \\
\hline Niagara-Haldimand-Norfolk & Nanticoke & 42.49 & -80.04 & 0.336 & 0.063 \\
\hline Greater Toronto Area & Medad & 43.22 & -79.53 & 0.066 & 0.024 \\
\hline & Grenadier & 43.38 & -79.28 & 0.143 & 0.046 \\
\hline Niagara-Haldimand-Norfolk & Windmill & 42.53 & -79.00 & 0.129 & 0.021 \\
\hline remote south-central Ontario & Plastic & 45.11 & -78.49 & 0.003 & 0.001 \\
\hline
\end{tabular}




$\begin{array}{rrrrrr}\text { Greater Montréal Area } & \text { West Croche } & 45.60 & -74.07 & 0.372 & 0.545 \\ & \text { Triton } & 45.59 & -74.05 & 0.019 & 0.584 \\ & \text { East Croche } & 45.60 & -74.03 & 0.002 & 0.763 \\ & \text { Hertel } & 45.33 & -73.91 & 0.002 & 0.722 \\ \text { Greater Trois-Rivières Area } & \text { Geai } & 45.60 & -73.60 & 0.088 & 0.025 \\ & \text { des bouleaux } & 45.33 & -73.19 & 0.002 & 0.801 \\ & \text { Francais } & 46.72 & -72.82 & 0.006 & 0.001 \\ \text { Greater Montréal Area } & \text { Gilron } & 46.72 & -72.82 & 0.029 & 0.004 \\ \text { Greater Québec City Area } & \text { Noir } & 46.72 & -72.82 & 0.016 & 0.004 \\ & \text { Gale } & 45.16 & -72.42 & 0.000 & 0.972 \\ \text { southern Québec } & \text { Clair } & 46.57 & -71.40 & 0.003 & 0.654 \\ \text { eastern Québec } & \text { Laflamme } & 47.94 & -71.07 & 0.031 & 0.190 \\ \text { Mejimkujik National Park } & \text { Megantic } & 45.34 & -70.53 & 0.026 & 0.000 \\ & \text { des Iles } & 48.32 & -69.91 & 0.008 & 0.001 \\ & \text { Cobrielle } & 44.18 & -65.21 & 0.014 & 0.002 \\ & \text { Peskowesk } & 44.19 & -65.17 & 0.011 & 0.001 \\ & \text { East Dam } & 44.27 & -65.16 & 0.018 & 0.003 \\ & \text { Bilsebois } & 52.39 & -64.53 & 0.018 & 0.001 \\ & \text { Hilchemakar } & 44.19 & -65.14 & 0.050 & 0.114\end{array}$

Table S6. Pearson coefficients of determination between sedimentation rates and $\mathrm{Hg}$ concentrations for each lake sediment core across Canada. Sites where relationships between sedimentation rates and $\mathrm{Hg}$ concentrations were significant are presented in bold.

\begin{tabular}{rrrrrr}
\multicolumn{1}{c}{ Region } & \multicolumn{1}{c}{ Lake site } & $\begin{array}{c}\text { Latitude } \\
\left({ }^{\circ} \mathbf{N}\right)\end{array}$ & $\begin{array}{c}\text { Longitude } \\
\left({ }^{\circ} \mathbf{W}\right)\end{array}$ & $\mathbf{r}^{\mathbf{2}}$ & p-value \\
\hline southern Yukon & Scout-1 & 60.79 & -135.43 & 0.34 & 0.17 \\
Prince of Wales Island & Control & 55.69 & -132.87 & 0.14 & 0.13 \\
& Mountain Goat & 55.65 & -132.67 & 0.00 & 0.75 \\
& Trumpeter & 55.88 & -132.63 & 0.28 & 0.04 \\
Trail British Columbia & Cabin & 55.65 & -132.50 & 0.34 & 0.17 \\
& Wilgress & 49.85 & -118.32 & 0.03 & 0.18 \\
& Nancy Greene & 49.15 & -117.57 & 0.01 & 0.51 \\
& Upper Little Slocan & $\mathbf{4 9 . 4 1}$ & $\mathbf{- 1 1 7 . 3 9}$ & $\mathbf{0 . 2 7}$ & $\mathbf{0 . 0 1}$ \\
& Summit & 50.89 & -117.37 & 0.01 & 0.39 \\
& Champion & 49.12 & -117.37 & 0.00 & 0.72 \\
& Bridal & $\mathbf{4 9 . 3 6}$ & $\mathbf{- 1 1 7 . 2 4}$ & $\mathbf{0 . 3 4}$ & $<\mathbf{0 . 0 1}$ \\
& Erie & $\mathbf{4 9 . 1 1}$ & $\mathbf{- 1 1 7 . 2 1}$ & $\mathbf{0 . 1 7}$ & $<\mathbf{0 . 0 1}$ \\
& Lomond & 49.04 & -117.19 & 0.01 & 0.39 \\
& Rosebud & 49.27 & -117.16 & 0.09 & 0.03 \\
& Hasse & 53.29 & -114.10 & $\mathbf{0 . 1 0}$ & $\mathbf{0 . 0 1}$ \\
& Cottonwood & $\mathbf{4 9 . 2 6}$ & $\mathbf{- 1 1 7 . 1 5}$ & 0.01 & 0.51 \\
& 53.38 & -114.45 & 0.24
\end{tabular}




$\begin{array}{rrr}\text { Pigeon } & \mathbf{5 3 . 0 2} & \mathbf{- 1 1 4 . 0 4} \\ 2014-Y & 56.82 & -113.27 \\ 2014-D & 56.95 & -113.03 \\ 2014-X & 57.36 & -112.73 \\ \text { RAMP464 } & 57.65 & -112.62 \\ \mathbf{2 0 1 4 - Z} & \mathbf{5 7 . 4 4} & \mathbf{- 1 1 2 . 5 3} \\ \text { RAMP175 } & 57.31 & -112.40 \\ \text { RAMP225 } & \mathbf{5 6 . 8 0} & \mathbf{- 1 1 1 . 9 2} \\ \text { RAMP223 } & \mathbf{5 6 . 7 8} & \mathbf{- 1 1 1 . 7 9} \\ \text { RAMP226 } & 56.81 & -111.72 \\ \text { NE20 } & 57.13 & -111.56 \\ \text { NE13 } & 57.07 & -111.48\end{array}$

$\begin{array}{lr}\mathbf{0 . 1 0} & \mathbf{0 . 0 1} \\ 0.01 & 0.55 \\ 0.11 & 0.03 \\ 0.04 & 0.12 \\ 0.01 & 0.45 \\ \mathbf{0 . 3 4} & <\mathbf{0 . 0 1} \\ 0.07 & 0.03 \\ \mathbf{0 . 6 8} & <\mathbf{0 . 0 1} \\ \mathbf{0 . 1 8} & \mathbf{0 . 0 1} \\ 0.06 & 0.18 \\ 0.07 & 0.13 \\ 0.03 & 0.32\end{array}$

$\begin{array}{lll}\text { RAMP418 } & \mathbf{5 7 . 2 9} & \mathbf{- 1 1 1 . 2 4}\end{array}$

0.13

0.01

Gregoire

56.48

$-111.15$

0.10

0.05

SE22

56.90

$-111.14$

0.28

$<0.01$

2014-C

$-110.95$

0.54

$<0.01$

RAMP268

$56.89-110.90$

RAMP185

$57.15 \quad-110.86$

0.56

$<0.01$

2014-B

$56.98-110.81$

RAMP209

$\mathbf{5 7 . 2 3}$

$-110.75$

0.75

$<0.01$

central Alberta

Wolf

54.41

$-110.55$

Athabasca Oil Sands Region

RAMP271

$56.64-110.22$

Flin Flon

Mclurg
Hamel

55.04

$-102.53$

0.52

0.59

$<0.01$

0.04

$<0.01$

0.23

0.13

0.07

$<0.01$

0.21

0.04

Meridian $\quad \mathbf{5 4 . 4 4} \quad \mathbf{- 1 0 1 . 5 8}$

0.44

$<0.01$

Douglas

$54.44 \quad-101.56$

Phantom

$54.44 \quad-101.52$

0.15

$<0.01$

0.23

$<0.01$

Cleaver $\quad \mathbf{5 4 . 4 2} \quad \mathbf{- 1 0 1 . 3 3}$

0.17

$<0.01$

0.40

$<0.01$

Nao $\quad 54.51 \quad-101.29$

Nekik $\quad 54.55 \quad-101.15$

0.34

$<0.01$

Louck

$54.40 \quad-100.44$

Thompson

remote Manitoba

TM-9 $\quad 55.45 \quad-98.38$

0.27

$<0.01$

TM-8

55.43

$-98.30$

$\begin{array}{lll}\text { TM-1 } & \mathbf{5 5 . 4 2} & \mathbf{- 9 7 . 3 9}\end{array}$

$\begin{array}{lll}\text { TM-3 } & \mathbf{5 5 . 4 6} & \mathbf{- 9 6 . 4 8}\end{array}$

$\begin{array}{lll}\mathrm{T} 1111 & 57.39 & -96.47\end{array}$

$\begin{array}{lll}\text { L442 } & 49.46 & \mathbf{- 9 3 . 4 9}\end{array}$

L373 $\quad 49.45 \quad-93.48$

0.10

$<0.01$

0.00

0.06

0.41

0.84

0.40

$<0.01$

0.01

$<0.01$

0.63

ELA

$\mathbf{4 9 . 4 2} \quad \mathbf{- 9 3 . 4 6}$

$\begin{array}{lll}\text { L337 } & \mathbf{4 9 . 4 3} & \mathbf{- 9 3 . 4 6}\end{array}$

L240 $49.39 \quad \mathbf{- 9 3 . 4 3}$

Nanticoke $\quad 42.49 \quad-80.04$
0.40

0.04

$<0.01$

0.12

0.42

$<0.01$

0.47

0.40

$<0.01$

0.00
$<0.01$

0.87 


\begin{tabular}{|c|c|c|c|c|c|}
\hline \multirow[t]{2}{*}{ Greater Toronto Area } & Medad & 43.22 & -79.53 & 0.00 & 0.71 \\
\hline & Grenadier & 43.38 & -79.28 & 0.03 & 0.21 \\
\hline Niagara-Haldimand-Norfolk & Windmill & 42.53 & -79.00 & 0.00 & 0.91 \\
\hline remote south-central Ontario & Plastic & 45.11 & -78.49 & 0.00 & 0.84 \\
\hline \multirow[t]{6}{*}{ Greater Montréal Area } & West Croche & 45.60 & -74.07 & 0.65 & $<0.01$ \\
\hline & Triton & 45.59 & -74.05 & 0.10 & 0.18 \\
\hline & East Croche & 45.60 & -74.03 & 0.01 & 0.36 \\
\hline & Hertel & 45.33 & -73.91 & 0.01 & 0.50 \\
\hline & Geai & 45.60 & -73.60 & 0.08 & 0.03 \\
\hline & des Bouleaux & 45.33 & -73.19 & 0.00 & 0.89 \\
\hline \multirow[t]{3}{*}{ Greater Trois-Rivières Area } & Francais & 46.72 & -72.82 & 0.07 & 0.32 \\
\hline & Gilron & 46.72 & -72.82 & 0.06 & 0.34 \\
\hline & Noir & 46.72 & -72.82 & 0.45 & 0.01 \\
\hline Greater Montréal Area & Gale & 45.16 & -72.42 & 0.03 & 0.24 \\
\hline \multirow[t]{2}{*}{ Greater Québec City Area } & Clair & 46.57 & -71.40 & 0.00 & 0.65 \\
\hline & Laflamme & 47.94 & -71.07 & 0.03 & 0.19 \\
\hline southern Québec & Megantic & 45.34 & -70.53 & 0.00 & 0.92 \\
\hline eastern Québec & des Iles & 48.32 & -69.91 & 0.34 & 0.01 \\
\hline \multirow[t]{4}{*}{ Kejimkujik National Park } & Cobrielle & 44.18 & -65.21 & 0.05 & 0.33 \\
\hline & Peskowesk & 44.19 & -65.17 & 0.00 & 0.71 \\
\hline & East Dam & 44.27 & -65.16 & 0.13 & 0.08 \\
\hline & Hilchemakaar & 44.19 & -65.14 & 0.02 & 0.36 \\
\hline western Québec & Brisebois & 52.39 & -64.53 & 0.06 & 0.07 \\
\hline
\end{tabular}

Table S7. Trends in post-1990 sedimentation rates for 18 of the 19 study regions across Canada as determined by Mann-Kendall (MK) analyses. A positive (>0) MK coefficient score represents an increasing trend while a negative $(<0)$ MK coefficient score represents a decreasing trend.

\begin{tabular}{rrrrrr} 
Region & Lake & MK coefficient & p-value & Latitude $\left({ }^{\circ} \mathbf{N}\right)$ & Longitude $\left({ }^{\circ} \mathbf{W}\right)$ \\
\hline \multirow{2}{*}{ Southern Yukon } & Scout-1 & -0.44 & 0.12 & 60.79 & -135.43 \\
& Control & -0.81 & 0.02 & 55.69 & -132.87 \\
& Mountain Goat & -0.75 & 0.00 & 55.65 & -132.67 \\
& Trumpeter & 0.33 & 0.37 & 55.88 & -132.63 \\
Trail British Columbia & Cabin & -0.67 & 0.31 & 55.65 & -132.50 \\
& Wilgress & -1.00 & 0.09 & 49.85 & -118.32 \\
& Nancy Greene & -0.02 & 1.00 & 49.15 & -117.57 \\
& Upper Little Slocan & +0.31 & 0.21 & 49.41 & -117.39 \\
& Summit & -0.16 & 0.59 & 50.89 & -117.37 \\
& Champion & -0.87 & 0.02 & 49.12 & -117.37 \\
& Bridal & -0.28 & 0.35 & 49.36 & -117.24 \\
& Erie & -0.78 & 0.00 & 49.11 & -117.21 \\
& Lomond & +0.38 & 0.12 & 49.04 & -117.19
\end{tabular}




\begin{tabular}{|c|c|c|c|c|c|}
\hline & Rosebud & -0.67 & 0.31 & 49.27 & -117.16 \\
\hline & Cottonwood & -0.43 & 0.17 & 49.26 & -117.15 \\
\hline \multirow[t]{2}{*}{ central Alberta } & Isle & -0.42 & 0.09 & 53.38 & -114.45 \\
\hline & Pigeon & -0.53 & 0.03 & 53.02 & -114.04 \\
\hline \multirow[t]{19}{*}{ Athabasca Oil Sands Region } & 2014-Y & -0.49 & 0.04 & 56.82 & -113.27 \\
\hline & 2014-D & +0.20 & 0.44 & 56.95 & -113.03 \\
\hline & 2014-X & -0.93 & 0.00 & 57.36 & -112.73 \\
\hline & RAMP 464 (L60) & -0.60 & 0.01 & 57.65 & -112.62 \\
\hline & $2014-Z$ & -0.86 & 0.00 & 57.44 & -112.53 \\
\hline & RAMP 175 (P13) & -0.75 & 0.00 & 57.31 & -112.40 \\
\hline & RAMP 225 & -0.71 & 0.00 & 56.80 & -111.92 \\
\hline & RAMP 223 (NW35) & +0.36 & 0.27 & 56.78 & -111.79 \\
\hline & RAMP226 (SW22) & -0.11 & 0.75 & 56.81 & -111.72 \\
\hline & NE20 & +0.33 & 0.37 & 57.13 & -111.56 \\
\hline & NE13 & -0.56 & 0.03 & 57.07 & -111.48 \\
\hline & RAMP 418 (Kearle) & -0.49 & 0.04 & 57.29 & -111.24 \\
\hline & Gregoire & -0.31 & 0.21 & 56.48 & -111.15 \\
\hline & SE22 & +0.81 & 0.02 & 56.90 & -111.14 \\
\hline & 2014-C & -0.13 & 0.64 & 56.39 & -110.95 \\
\hline & RAMP 268 (E15) & -0.64 & 0.01 & 56.89 & -110.90 \\
\hline & RAMP 185 & -0.53 & 0.03 & 57.15 & -110.86 \\
\hline & 2014-B & -0.02 & 1.00 & 56.98 & -110.81 \\
\hline & RAMP 209 (P7) & -0.71 & 0.00 & 57.23 & -110.75 \\
\hline central Alberta & Wolf & +0.20 & 0.44 & 54.41 & -110.55 \\
\hline Athabasca Oil Sands Region & RAMP 271 & -0.96 & 0.00 & 56.64 & -110.22 \\
\hline \multirow[t]{9}{*}{ Flin Flon } & Mclurg & -0.20 & 0.44 & 55.04 & -102.53 \\
\hline & Hamel & +0.22 & 0.47 & 54.49 & -101.58 \\
\hline & Meridian & -0.20 & 0.44 & 54.44 & -101.58 \\
\hline & Douglas & +0.49 & 0.04 & 54.44 & -101.56 \\
\hline & Phantom & -0.64 & 0.01 & 54.44 & -101.52 \\
\hline & Cleaver & -0.42 & 0.09 & 54.42 & -101.33 \\
\hline & Naosap Mud & +0.75 & 0.00 & 54.51 & -101.29 \\
\hline & Nekik & -0.24 & 0.35 & 54.55 & -101.15 \\
\hline & Loucks & +0.38 & 0.12 & 54.40 & -100.44 \\
\hline \multirow[t]{4}{*}{ Thompson } & TM-9A & -0.60 & 0.01 & 55.45 & -98.38 \\
\hline & TM-8A & +0.38 & 0.12 & 55.43 & -98.30 \\
\hline & TM-1A & +0.07 & 0.90 & 55.42 & -97.39 \\
\hline & TM-3A & +0.44 & 0.12 & 55.46 & -96.48 \\
\hline remote Manitoba & $\mathrm{T} 1111$ & -0.14 & 0.14 & 57.39 & -96.47 \\
\hline \multirow[t]{4}{*}{ ELA } & L442 & +0.24 & 0.35 & 49.46 & -93.49 \\
\hline & L373 & +0.33 & 0.37 & 49.45 & -93.48 \\
\hline & L378 & +0.71 & 0.00 & 49.42 & -93.46 \\
\hline & L337 & +0.56 & 0.03 & 49.43 & -93.46 \\
\hline
\end{tabular}




$\begin{array}{rrrrrr} & \text { L240 } & -0.02 & 1.00 & 49.39 & -93.43 \\ \text { Niagara-Haldimand-Norfolk } & \text { Nanticoke } & +0.46 & 0.06 & 42.49 & -80.04 \\ \text { Greater Toronto Area } & \text { Medad } & -0.64 & 0.01 & 43.22 & -79.53 \\ & \text { Grenadier } & -0.64 & 0.01 & 43.38 & -79.28 \\ \text { Niagara-Haldimand-Norfolk } & \text { Windmill Point Pond } & +0.05 & 0.88 & 42.53 & -79.00 \\ \text { remote south-central Ontario } & \text { Plastic } & -1.00 & 0.03 & 45.11 & -78.49 \\ \text { Greater Montréal Area } & \text { West Croche } & +0.02 & 1.00 & 45.60 & -74.07 \\ & \text { Triton } & +0.60 & 0.01 & 45.59 & -74.05 \\ & \text { East Croche } & +0.24 & 0.35 & 45.60 & -74.03 \\ \text { Hreater Trois Rivières Area } & \text { Hertel } & +0.46 & 0.06 & 45.33 & -73.91 \\ \text { Greater Montréal Area } & \text { Geai } & +0.27 & 0.28 & 45.60 & -73.60 \\ \text { Greater Québec City Area } & \text { des Bouleaux } & +0.27 & 0.28 & 45.33 & -73.19 \\ \text { eastern Québec } & \text { Giron } & -1.00 & 0.03 & 46.72 & -72.82 \\ \text { wejimkujik National Park } & \text { Gale } & +0.16 & 0.53 & 45.16 & -72.42 \\ & \text { Laflamme } & +0.06 & 0.92 & 47.94 & -71.07 \\ & \text { des Iles } & +0.33 & 0.45 & 48.32 & -69.91 \\ & \text { Cobrielle } & +1.00 & 0.09 & 44.18 & -65.21 \\ & \text { Peskowesk } & -0.17 & 0.60 & 44.19 & -65.17 \\ & \text { East Dam } & +1.00 & 0.01 & 44.27 & -65.16 \\ & \text { Hilchemakaar } & +0.24 & 0.55 & 44.19 & -65.14 \\ & \text { Brisbois } & +0.38 & 0.12 & 52.39 & -64.53\end{array}$

Table S8. PCA loading scores for average anthropogenic post-2005 anthropogenic Hg flux $\left(\mathrm{HgF}_{\mathrm{FF}, \mathrm{CA}}\right)$ and $\mathrm{Hg}$ flux ratio with environmental factors.

Environmental factors

PC1

PC2

\begin{tabular}{|c|c|c|c|}
\hline \multirow{9}{*}{ Hg flux } & Latitude & 30.34 & 2.03 \\
\hline & Longitude & 25.46 & 3.45 \\
\hline & Absence/presence of point source & 7.18 & 34.44 \\
\hline & Distance to closest source & 1.12 & 46.83 \\
\hline & Elevation & 4.92 & 0.37 \\
\hline & Lake Area & 2.93 & 0.50 \\
\hline & Catchment Area & 2.94 & 0.74 \\
\hline & Catchment Area: Lake Area ratio & 23.83 & 5.86 \\
\hline & Precipitation & 1.09 & 1.27 \\
\hline \multirow{6}{*}{ Hg ratio } & Latitude & 22.24 & 4.55 \\
\hline & Longitude & 23.06 & 5.45 \\
\hline & Absence/presence of point source & 8.20 & 28.48 \\
\hline & Distance to closest source & 1.36 & 43.65 \\
\hline & Elevation & 9.60 & 4.96 \\
\hline & Lake Area & 2.92 & 0.06 \\
\hline
\end{tabular}




$\begin{array}{rrr}\text { Catchment Area } & 1.88 & 2.12 \\ \text { Catchment Area: Lake Area ratio } & 2.47 & 4.19 \\ \text { Precipitation } & 23.14 & 6.06\end{array}$

Table S9. Mann-Kendall (MK) coefficient values from trend analysis on wet deposition $\mathrm{Hg}$ fluxes from mercury deposition network (MDN) monitoring stations located within $100 \mathrm{~km}$ of sediment sampling sites including Kejimkujik National Park (NS), Marcell Experiment Forest (MN-16) and Fernberg (MN-18) in the Experimental Lakes Area (ELA) of northwestern Ontario, Egbert in South-central Ontario and St. Anicet southwest of Montréal (QC). ${ }^{12-14}$

\begin{tabular}{rrrrrrr}
\multicolumn{1}{c}{ Region } & \multicolumn{1}{c}{ Station } & $\begin{array}{c}\text { Latitude } \\
\left({ }^{\mathbf{}} \mathbf{N}\right)\end{array}$ & $\begin{array}{c}\text { Longitude } \\
\left({ }^{\circ} \mathbf{W}\right)\end{array}$ & Time period & $\begin{array}{c}\text { MK } \\
\text { coefficient }\end{array}$ & p-value \\
\hline Nova Scotia & Kejimkujik & 44.43 & -65.20 & $1997-2018$ & -0.11 & 0.50 \\
South-central Ontario & Egbert & 44.23 & -79.79 & $2001-2018$ & 0.11 & 0.75 \\
Greater Montreal Area & St Anicet & 45.12 & -74.29 & $1999-2006$ & 0.14 & 0.71 \\
northern Minnesota & MN16 (ELA) & 47.53 & -93.46 & $1997-2018$ & -0.23 & 0.13 \\
northern Minnesota & MN18 (ELA) & 47.94 & -91.49 & $1998-2017$ & -0.41 & 0.02 \\
north-western Ontario & ELA & 49.46 & -93.49 & $1992-2010$ & -0.02 & 0.92
\end{tabular}

Table S10. Post-2005 sedimentary anthropogenic $\mathrm{Hg}$ flux $\left(\mathrm{HgF}_{\mathrm{FF}, \mathrm{CA}}\right)$, modelled 2015 total $\mathrm{Hg}$ deposition (= wet + dry), modelled 2015 wet deposition to the entire lake catchment, modelled $2015 \mathrm{Hg}$ deposition to the lake surface using wet deposition for summertime and wet + dry deposition (snow) for wintertime, and sediment: modelled wet+ dry deposition to the lake surface $\mathrm{Hg}$ flux ratio for each lake sediment core site across Canada.

\begin{tabular}{|c|c|c|c|c|c|c|}
\hline Region & Lake & $\begin{array}{c}\text { Sediment- } \\
\text { derived post- } \\
2005 \mathrm{Hg} \text { flux }^{-} \\
\left(\mu \mathrm{g} \mathrm{m}^{-2} \mathrm{yr}^{-1}\right)\end{array}$ & $\begin{array}{c}\text { Modelled } \\
\text { total } \\
\text { deposition } \\
\left(\mu \mathrm{g} \mathrm{m}^{-2} \mathrm{yr}^{-1}\right)\end{array}$ & $\begin{array}{c}\text { Modelled Wet } \\
\text { deposition to } \\
\text { lake } \\
\text { catchment } \\
\left(\mu \mathrm{g} \mathrm{m}^{-2} \mathrm{yr}^{-1}\right)\end{array}$ & $\begin{array}{c}\text { Modelled } \\
\text { Wet + Dry } \\
\text { deposition } \\
\text { to lake } \\
\text { surface } \\
\left(\mu \mathbf{g ~ m}^{-2} \mathbf{y r}^{-1}\right)\end{array}$ & $\begin{array}{c}\text { Sediment: } \\
\text { modelled } \\
\text { Wet + Dry } \\
\text { deposition to } \\
\text { lake surface } \\
\text { ratio }\end{array}$ \\
\hline
\end{tabular}

\begin{tabular}{lrllllll}
\hline NS & Cobrielle & 12.68 & 21.81 & 6.81 & 10.22 & 1.24 & 1.88 \\
NS & East Dam & 19.84 & 22.94 & 7.27 & 10.56 & 8.93 & 1.26 \\
NS & Hilchemakaar & 11.29 & 16.44 & 6.53 & 6.62 & 9.44 & 1.54 \\
NS & Peskowesk & 14.51 & 18.56 & 30.05 & 22.23 & 21.95 & 0.23 \\
BC & Champion & 5.08 & 15.06 & 8.81 & 8.67 & 0.07 \\
BC & Cottonwood & 0.58 & 16.45 & 9.31 & 9.30 & 0.42 \\
BC & Erie & 3.91 & & & &
\end{tabular}




\begin{tabular}{|c|c|c|c|c|c|c|}
\hline $\mathrm{BC}$ & Rosebud & 3.99 & 14.24 & 7.88 & 7.78 & 0.51 \\
\hline $\mathrm{BC}$ & Lomond & 11.35 & 20.40 & 13.33 & 13.19 & 0.86 \\
\hline $\mathrm{BC}$ & Nancy Greene & 17.94 & 16.73 & 7.84 & 8.38 & 2.14 \\
\hline $\mathrm{BC}$ & Bridal & 12.50 & 12.13 & 5.12 & 5.06 & 2.47 \\
\hline $\mathrm{BC}$ & Wilgress & 7.75 & 13.14 & 6.70 & 6.60 & 1.17 \\
\hline $\mathrm{BC}$ & Summit & 7.87 & 13.74 & 11.14 & 10.37 & 0.76 \\
\hline $\mathrm{BC}$ & Upper Little Slocan & 6.56 & 20.44 & 14.83 & 14.15 & 0.46 \\
\hline $\mathrm{ON}$ & Plastic & 3.28 & 20.53 & 7.99 & 10.01 & 0.33 \\
\hline NHN & Nanticoke & 7.19 & 12.76 & 10.82 & 11.30 & 0.64 \\
\hline NHN & Windmill Point Pond & 408.82 & 25.44 & 11.10 & 13.35 & 30.63 \\
\hline GTA & Medad & 94.83 & 26.42 & 8.15 & 12.14 & 7.81 \\
\hline GTA & Grenadier & 46.18 & 11.02 & 9.61 & 9.94 & 4.64 \\
\hline ELA & L442 & 4.83 & 18.57 & 6.92 & 8.14 & 0.59 \\
\hline ELA & L337 & 7.97 & 18.75 & 7.45 & 8.62 & 0.92 \\
\hline ELA & L373 & 5.32 & 18.67 & 7.13 & 8.33 & 0.64 \\
\hline ELA & L240 & 9.42 & 18.89 & 7.68 & 8.82 & 1.07 \\
\hline ELA & L378 & 5.80 & 18.86 & 7.54 & 8.71 & 0.67 \\
\hline GMC & West Croche & 16.64 & 34.11 & 8.18 & 12.03 & 1.38 \\
\hline GMC & East Croche & 15.02 & 34.60 & 8.20 & 12.16 & 1.24 \\
\hline GMC & Triton & 26.54 & 34.42 & 8.21 & 12.12 & 2.19 \\
\hline WQC & Brisebois & 16.80 & 19.07 & 7.36 & 9.02 & 1.86 \\
\hline GMC & Geai & 79.76 & 22.36 & 9.97 & 11.93 & 6.69 \\
\hline GMC & Hertel & 50.10 & 18.89 & 7.40 & 9.41 & 5.33 \\
\hline EQC & des Iles & 2.24 & 18.49 & 8.07 & 9.24 & 0.24 \\
\hline $\mathrm{TH}$ & TM-1A & 16.13 & 17.63 & 4.71 & 5.47 & 2.95 \\
\hline $\mathrm{TH}$ & TM-8A & 16.29 & 15.27 & 4.80 & 5.31 & 3.07 \\
\hline $\mathrm{TH}$ & TM-9A & 7.19 & 16.44 & 4.79 & 5.36 & 1.34 \\
\hline $\mathrm{TH}$ & TM-3A & 8.72 & 16.23 & 4.76 & 4.96 & 1.76 \\
\hline $\mathrm{FF}$ & Nekik & 22.70 & 18.18 & 4.58 & 5.42 & 4.19 \\
\hline $\mathrm{FF}$ & Douglas & 947.71 & 15.44 & 4.42 & 5.14 & 184.43 \\
\hline $\mathrm{FF}$ & Louck & 18.77 & 15.31 & 4.75 & 5.32 & 3.53 \\
\hline $\mathrm{FF}$ & Phantom & 1398.15 & 15.25 & 4.44 & 5.11 & 273.53 \\
\hline $\mathrm{FF}$ & Mclurg & 18.45 & 17.11 & 4.33 & 5.02 & 3.68 \\
\hline $\mathrm{FF}$ & Cleaver & 159.55 & 16.03 & 4.77 & 5.37 & 29.70 \\
\hline $\mathrm{FF}$ & Nao & 68.04 & 16.76 & 4.80 & 5.41 & 12.58 \\
\hline FF & Hamel & 1113.19 & 15.25 & 4.39 & 5.19 & 214.62 \\
\hline $\mathrm{FF}$ & Meridian & 648.81 & 15.53 & 4.40 & 5.15 & 126.01 \\
\hline AOSR & NE13 & 0.84 & 14.34 & 4.27 & 4.74 & 0.18 \\
\hline AOSR & NE20 & 14.88 & 14.69 & 3.90 & 4.43 & 3.36 \\
\hline AOSR & SE22 & 11.35 & 15.52 & 4.90 & 5.52 & 2.06 \\
\hline AOSR & RAMP226 (SW22) & 34.00 & 14.27 & 3.68 & 4.59 & 7.41 \\
\hline AOSR & RAMP 223 (NW35) & 6.36 & 14.89 & 3.80 & 4.82 & 1.32 \\
\hline AOSR & RAMP 268 (E15) & 6.28 & 16.76 & 4.01 & 4.74 & 1.32 \\
\hline
\end{tabular}




\begin{tabular}{|c|c|c|c|c|c|c|}
\hline AOSR & RAMP 418 (Kearle) & 4.92 & 13.07 & 3.76 & 4.36 & 1.13 \\
\hline AOSR & RAMP 225 & 4.42 & 15.12 & 3.83 & 4.93 & 0.90 \\
\hline AOSR & RAMP 185 & 18.64 & 14.39 & 4.05 & 5.00 & 3.73 \\
\hline AOSR & 2014-B & 7.11 & 14.20 & 4.42 & 4.90 & 1.45 \\
\hline AOSR & RAMP 209 (P7) & 12.24 & 14.06 & 3.77 & 4.72 & 2.59 \\
\hline AOSR & Gregoire & 26.13 & 15.93 & 3.65 & 5.04 & 5.18 \\
\hline AOSR & RAMP 175 (P13) & 14.45 & 15.52 & 3.81 & 3.86 & 3.74 \\
\hline AOSR & 2014-C & 6.68 & 14.08 & 3.65 & 4.80 & 1.39 \\
\hline AOSR & 2014-Z & 12.12 & 13.77 & 3.32 & 3.34 & 3.62 \\
\hline AOSR & 2014-X & 7.97 & 14.06 & 3.46 & 3.49 & 2.28 \\
\hline AOSR & RAMP 271 & 1.57 & 12.71 & 3.56 & 3.99 & 0.39 \\
\hline AOSR & 2014-D & 16.12 & 12.71 & 3.02 & 3.49 & 4.62 \\
\hline AOSR & RAMP 464 (L60) & 10.39 & 14.44 & 3.46 & 3.63 & 2.86 \\
\hline AOSR & 2014-Y & 18.20 & 13.01 & 3.09 & 3.55 & 5.13 \\
\hline $\mathrm{AB}$ & Pigeon & 5.87 & 26.11 & 5.66 & 7.98 & 0.74 \\
\hline $\mathrm{AB}$ & Wolf & 3.17 & 15.51 & 4.31 & 4.79 & 0.66 \\
\hline $\mathrm{AB}$ & Isle & 5.95 & 32.23 & 7.17 & 7.79 & 0.76 \\
\hline POW & Trumpeter & 7.49 & 28.28 & 8.37 & 12.65 & 0.59 \\
\hline POW & Mountain Goat & 9.01 & 24.66 & 6.41 & 9.71 & 0.93 \\
\hline POW & Control & 8.74 & 27.93 & 8.67 & 11.92 & 0.73 \\
\hline POW & Cabin & 5.17 & 28.23 & 6.45 & 11.57 & 0.45 \\
\hline GTR & Francais & 3.01 & 19.56 & 6.62 & 8.17 & 0.37 \\
\hline GTR & Giron & 8.95 & 19.56 & 6.62 & 8.17 & 1.09 \\
\hline GTR & Noir & 6.99 & 19.56 & 6.62 & 8.17 & 0.86 \\
\hline YK & Scout-1 & 0.34 & 11.67 & 3.09 & 2.96 & 0.11 \\
\hline $\mathrm{AB}$ & Hasse & 0.00 & 34.78 & 6.17 & 7.99 & 0.00 \\
\hline GMC & des Bouleaux & 31.43 & 18.92 & 8.06 & 9.82 & 3.20 \\
\hline GMC & Gale & 22.78 & 19.53 & 11.04 & 12.14 & 1.88 \\
\hline SQ & Megantic & 5.90 & 17.17 & 8.30 & 9.40 & 0.63 \\
\hline $\mathrm{MB}$ & T1111 & 2.00 & 14.21 & 4.75 & 4.53 & 0.44 \\
\hline GQC & Laflamme & 27.77 & 16.06 & 7.24 & 8.01 & 3.47 \\
\hline GQC & Clair & 15.68 & 21.85 & 7.03 & 9.18 & 1.71 \\
\hline
\end{tabular}

